ARTICLE

\title{
ARIH1 signaling promotes anti-tumor immunity by targeting PD-L1 for proteasomal degradation
}

Youqian Wu ${ }^{1,11}$, Chao Zhang 2,3,11, Xiaolan Liu¹, ${ }^{1,1}$ Zhengfu He ${ }^{4,11}$, Bing Shan ${ }^{5}$, Qingxin Zeng ${ }^{4}$, Qingwei Zhao ${ }^{1}$ Huaying Zhu ${ }^{6}$, Hongwei Liao ${ }^{7}$, Xufeng Cen ${ }^{1}$, Xiaoyan Xu' ${ }^{1}$, Mengmeng Zhang ${ }^{5}$, Tingjun Hou ${ }^{8}{ }^{8}$, Zhe Wang ${ }^{8}$, Huanhuan Yan ${ }^{1}$, Shuying Yang ${ }^{1}$, Yaqin Sun ${ }^{1}$, Yanying Chen ${ }^{1}$, Ronghai Wu', Tingxue Xie ${ }^{1}$, Wei Chen (D) ${ }^{6}$, Ayaz Najafov (1) 9,12凶, Songmin Ying (1) ${ }^{2,7,12 凶}$ \& Hongguang Xia (1) 1,10,12凶

Cancer expression of PD-L1 suppresses anti-tumor immunity. PD-L1 has emerged as a remarkable therapeutic target. However, the regulation of PD-L1 degradation is not understood. Here, we identify several compounds as inducers of PD-L1 degradation using a highthroughput drug screen. We find EGFR inhibitors promote PD-L1 ubiquitination and proteasomal degradation following GSK3 $\alpha$-mediated phosphorylation of Ser279/Ser283. We identify ARIH1 as the E3 ubiquitin ligase responsible for targeting PD-L1 to degradation. Overexpression of ARIH1 suppresses tumor growth and promotes cytotoxic $\mathrm{T}$ cell activation in wild-type, but not in immunocompromised mice, highlighting the role of ARIH1 in antitumor immunity. Moreover, combining EGFR inhibitor ES-072 with anti-CTLA4 immunotherapy results in an additive effect on both tumor growth and cytotoxic $\mathrm{T}$ cell activation. Our results delineate a mechanism of PD-L1 degradation and cancer escape from immunity via EGFR-GSK3 $\alpha$-ARIH1 signaling and suggest GSK3 $\alpha$ and ARIH1 might be potential drug targets to boost anti-tumor immunity and enhance immunotherapies.

\footnotetext{
${ }^{1}$ Department of Biochemistry and Research Center of Clinical Pharmacy of The First Affiliated Hospital, Zhejiang University School of Medicine, Hangzhou 310058, China. ${ }^{2}$ International Institutes of Medicine, The Fourth Affiliated Hospital of Zhejiang University School of Medicine, Yiwu 322000, China. ${ }^{3}$ Key Laboratory Respiratory Disease of Zhejiang Province, Department of Anatomy and Department of Respiratory and Critical Care Medicine of the Second Affiliated Hospital, Zhejiang University School of Medicine, Hangzhou, Zhejiang 310009, China. ${ }^{4}$ Department of Thoracic Surgery, Sir Run Run Shaw Hospital, School of Medicine, Zhejiang University, Hangzhou, Zhejiang 310016, China. ${ }^{5}$ Interdisciplinary Research Center on Biology and Chemistry, Shanghai Institute of Organic Chemistry, Chinese Academy of Sciences, Shanghai 201203, China. ${ }^{6}$ Department of Cell Biology and Department of Cardiology of the Second Affiliated Hospital, Zhejiang University School of Medicine, Hangzhou 310058, China. ${ }^{7}$ Key Laboratory Respiratory Disease of Zhejiang Province, Department of Pharmacology and Department of Respiratory and Critical Care Medicine of the Second Affiliated Hospital, Zhejiang University School of Medicine, Hangzhou, Zhejiang 310009, China. ${ }^{8}$ Hangzhou Institute of Innovative Medicine, College of Pharmaceutical Sciences, Zhejiang University, Hangzhou 310058, China. ${ }^{9}$ Department of Cell Biology, Harvard Medical School, Boston, MA 02115, USA. ${ }^{10}$ Liangzhu Laboratory, Zhejiang University Medical Center, Hangzhou 311121, China. ${ }^{11}$ These authors contributed equally: Youqian Wu, Chao Zhang, Xiaolan Liu, Zhengfu He. ${ }^{12}$ These authors jointly

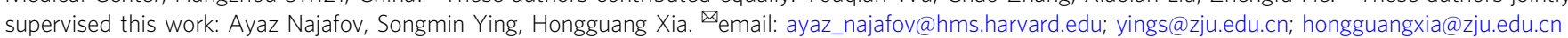


P rogrammed death ligand-1 (PD-L1) is constitutively expressed on the surface of cancer cells ${ }^{1-4}$. The interaction between PD-L1 and its receptor, programmed death protein-1 (PD-1), which is mainly expressed on the surface of $\mathrm{T}$ cells, results in cancer cell evasion from immune surveillance ${ }^{5}$. PD-1 and PD-L1 have become important immune checkpoint blockade immunotherapy targets for different types of cancers ${ }^{5,6}$. However, resistance to such immunotherapy approaches is prevalent and the mechanisms of resistance are not well understood.

Proteasomal degradation of $\mathrm{PD}-\mathrm{L} 1$ has been reported to be promoted by cyclin D-CDK4-mediated phosphorylation followed by Cullin $3^{\text {SPOP }}$-dependent ubiquitination ${ }^{7}$, as well as glycogen synthase kinase 3- $\beta$ (GSK3 $\beta$ )-mediated phosphorylation followed by $\beta$-TrCP-dependent ubiquitination ${ }^{8}$. However, the dynamic ubiquitin modification of PD-L1 for proteasomal degradation should mostly occur in the intracellular segment of PD-L1, which is not addressed before. Ariadne-1 homolog (ARIH1) is a member of the Ariadne family of E3 ubiquitin ligases with a cognate E2 enzyme UBCH7 ${ }^{9-11}$. ARIH1 is known to play a role in protein translation regulation in response to DNA damage and to ubiquitinate EIF4E2 ${ }^{12}$. The role of ARIH1 in PD-L1 degradation or anti-tumor immunity is not known.

Epidermal growth factor receptor (EGFR) is a major receptor tyrosine kinase, frequently overactivated in cancers, with established roles in cell growth and survival ${ }^{13-15}$. Patients with EGFRmutant-driven tumors develop resistance to EGFR tyrosine kinase inhibitor treatments, which are usually accompanied by the acquisition of EGFR mutations ${ }^{16}$. Clinical retrospectives suggest that EGFR mutations are associated with low response rates to immune therapies in non-small cell lung cancer ${ }^{17,18}$. Importantly, EGFR mutations are associated with increased PDL1 expression ${ }^{19-21}$. EGFR activation promotes PD-L1 expression via Janus kinase/signal transducer and activator of transcription 3 (JAK/STAT3) signaling pathway ${ }^{22}$ and inhibits its degradation via phosphorylation mediated by GSK3 $\beta^{8}$ in the extracellular part of PD-L1, contributing to cancer escape from anti-tumor immunity. These findings strongly indicate that EGFR works as a critical regulator of tumor immune surveillance via regulation of PD-L1 expression, but the mechanism of this regulation is not fully understood, especially as the reported phosphorylation sites are in the extracellular part of PD-L1.

In this study, we screened a panel of 2125 Food and Drug Administration (FDA)-approved drugs or drug candidates and found that ES-072, a third-generation EGFR inhibitor, induced a potent degradation of PD-L1. Our mechanistic findings suggest that inhibition of EGFR activates GSK3 $\alpha$ by suppressing AKT activity, which subsequently promotes the phosphorylation at the intracellular Ser279 and Ser283 residues of PD-L1, leading to ARIH1-mediated ubiquitination and proteasome-mediated degradation. Cancer-associated mutation of ARIH1 compromises ubiquitination of PD-L1. Together, our results suggest ARIH1 is the E3 ligase for PD-L1, which could lead to the development of therapeutic strategies to overcome immunotherapy resistance in cancers and enhance checkpoint blockade therapy efficacy.

\section{Results}

A high-throughput screen of 2125 FDA-approved drugs or drug candidates identifies promoters of PD-L1 degradation. To establish a fluorescence-based high-throughput screening protocol for the membrane levels of endogenous PD-L1, we used a phycoerythrin-conjugated anti-PD-L1 antibody and interferon- $\gamma$ (IFN $\gamma$ )-treated U937 cells (histiocytic lymphosarcoma cell line). IFN $\gamma$ enhances the basal expression levels of PD-L1, allowing for a wider dynamic range and, thus, a better screening system ${ }^{23,24}$.
As a positive control for drug-induced PD-L1 level decrease, we used Ruxolitinib, a JAK1/2 inhibitor ${ }^{25}$ (Supplementary Fig. 1a). As expected, PD-L1 levels were induced by IFN $\gamma$ and blocked by Ruxolitinib treatment (Supplementary Fig. 1b-d).

Out of 2125 FDA-approved drugs or drug candidates screened, 160 were found to reduce the membranal PD-L1 levels (Supplementary Fig. 1e and Supplementary Table 1). We classified the positive hits according to the signaling pathways they were known to be involved in, which included the JAK/ STAT pathway, the phosphatidylinositol 3-kinase (PI3K)/AKT/ mammalian target of the rapamycin pathway, pathways that regulate the cell cycle, and protein tyrosine kinases (Fig. 1a). The screen confirmed the existing knowledge about PD-L1 regulation, as 13 different JAK inhibitors were found to decrease plasma membrane PD-L1 levels by more than $25 \%$; this also indicated that the screen setup is valid, as JAK was the top drug target inhibition of which resulted in the PD-L1 level decrease.

On the other hand, EGFR has also been strongly linked to PD-L1 protein level regulation. From the EGFR inhibitors that promote PD-L1 level decrease, AZD9291 and ES-072 were selected for our screen follow-up and mechanistic studies to investigate what drives PD-L1 degradation downstream of EGFR inhibition. AZD9291, Osimertinib, is a potent and selective mutant EGFR (L858R/ T790M) inhibitor, which is widely used in clinic $^{26,27}$. ES-072 is a third-generation EGFR inhibitor designed by our team to overcome drug-resistance-induced EGFR mutation L858R/T790M. Confirming the screen findings, both ES-072 and AZD9291 dramatically reduced the IFN $\gamma$-induced membranal PD-L1 levels in U937 cells following treatment with the compounds, as judged by western blotting and flow cytometry results (Fig. 1b-d). The effect of the EGFR inhibitors on PD-L1 levels was time-dependent, reaching a plateau following $12 \mathrm{~h}$ of treatment (Fig. 1e-g). Similar findings were seen in H1975 cells (Supplementary Fig. 2a-d) and interleukin-4 (IL-4)-treated peritoneal-derived macrophages (PDMs) (Supplementary Fig. 2e-g).

EGFR inhibitors are known to promote PD-L1 degradation via the GSK3 $\alpha / \beta-\operatorname{TrCP}$ pathway $^{8}$. Surprisingly, contrary to the previous reports, we found that knockdown of $\beta$-TrCP did not fully block the reduction of PD-L1 levels induced following EGFR inhibition (Fig. 1h, i), whereas GSK3 $\alpha / \beta$ inhibitor LY2090314 did block this reduction (Fig. 1j-1). LY2090314 was found to be highly specific for GSK3 $\alpha$ with an half maximal inhibitory concentration (IC50) value of $0.87 \pm 0.09 \mathrm{nM}$ (Supplementary Table 2). These results indicated that a $\beta$-TrCP-independent, yet GSK $3 \alpha / \beta$ dependent mechanism regulating PD-L1 levels downstream of EGFR should exist.

ES-072 promotes proteasomal PD-L1 degradation via EGFR inhibition. The rest of our studies were continued using ES-072 due to its stability and specificity (Supplementary Table 3). As, upon EGFR inhibition, PD-L1 has been shown to be degraded via the proteasome, we first determined whether ES-072 treatment also results in a proteasome-dependent degradation of PD-L1. Both western blotting and flow cytometry analysis of plasma membrane PD-L1 levels confirmed that EGFR inhibition results in a proteasome-mediated PD-L1 degradation, as the decrease in the PD-L1 levels induced by ES-072 was rescued by the proteasome inhibitor MG132 (Supplementary Fig. 3a-c).

In accord with previous reports ${ }^{28,29}$, EGF treatment promoted an increase of PD-L1 levels (Supplementary Fig. 3d-f), whereas small interfering RNA (siRNA)-mediated knockdown of EGFR decreased PD-L1 levels (Supplementary Fig. 3g). ES-072 was found to be highly specific for EGFR in an in vitro kinase-profiling screen, where it was found to inhibit wild-type, 

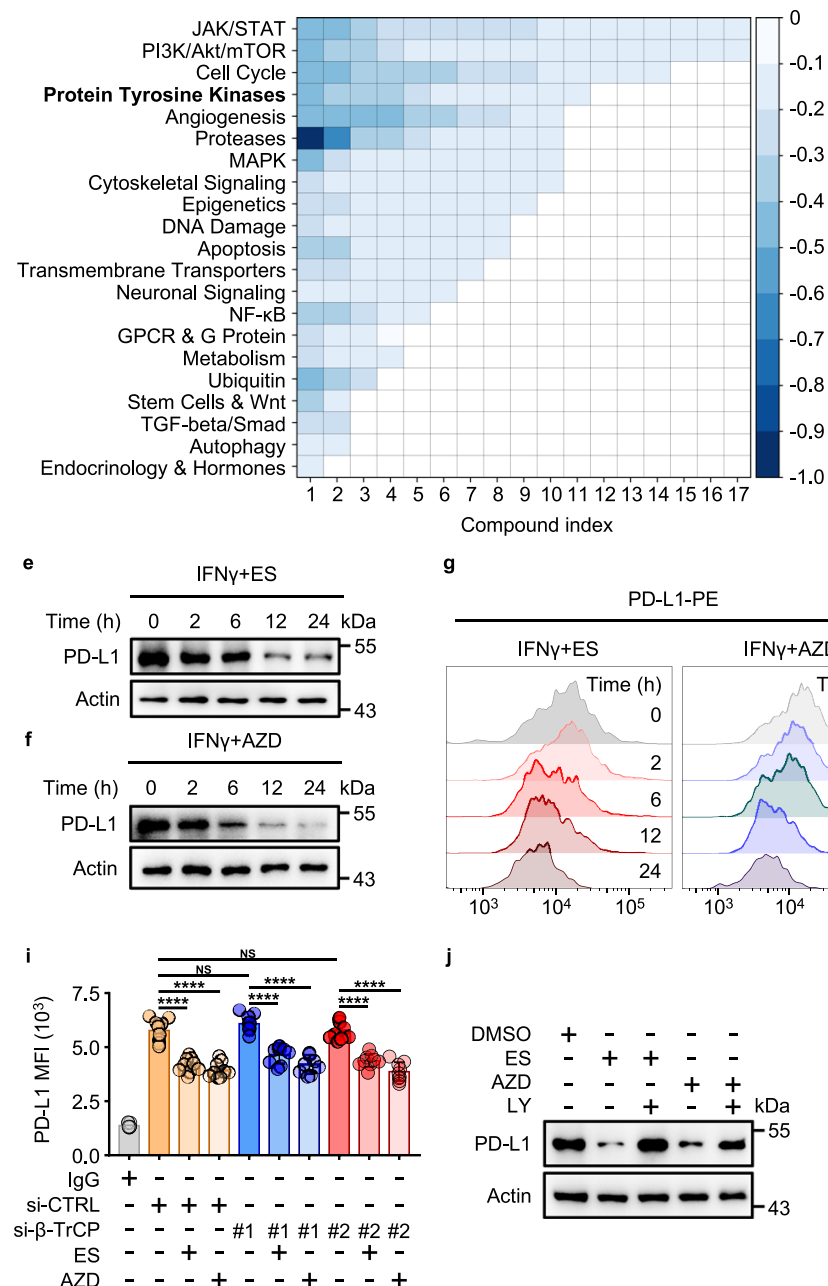

g

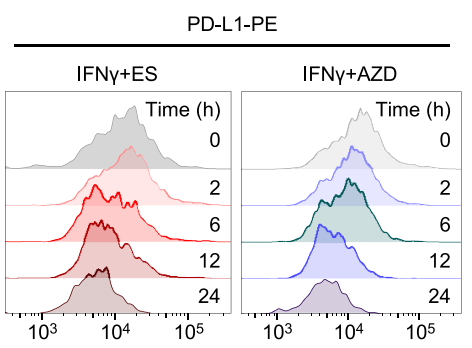

j

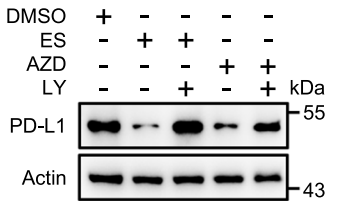

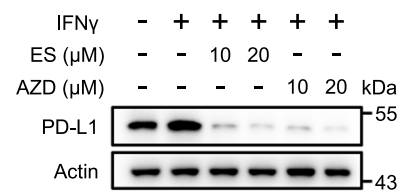
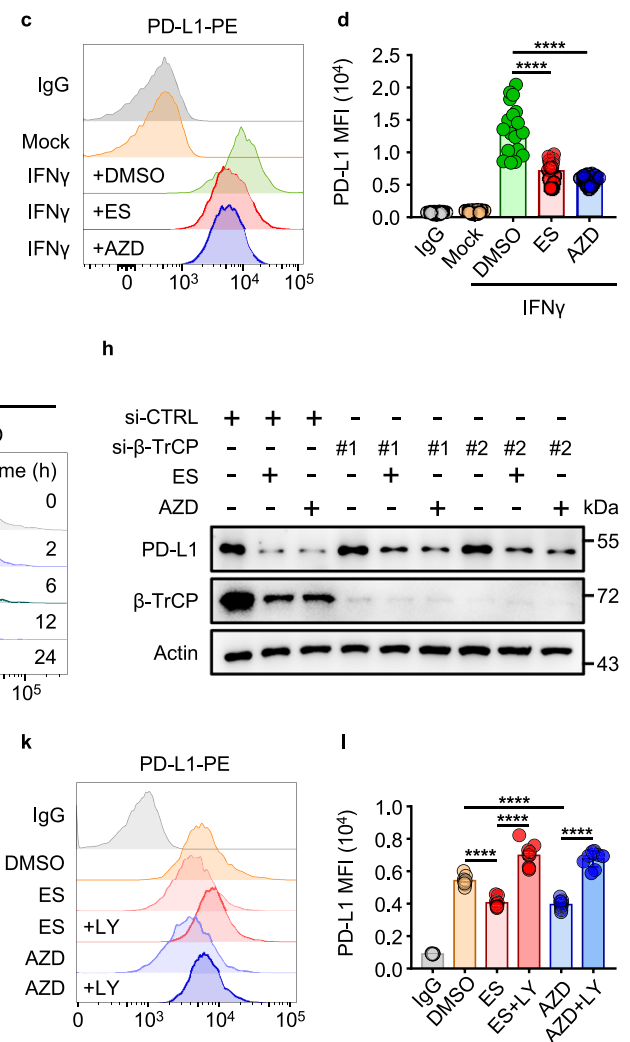

Fig. 1 EGFR inhibitors were screened to reduce membrane PD-L1 levels. a High-throughput screening of 2125 FDA-approved drugs or drug candidates. U937 cells were incubated with IFN $\gamma(100 \mathrm{ng} / \mathrm{mL}$ ) for $48 \mathrm{~h}$, treated with the drugs at $10 \mu \mathrm{M}$ for $12 \mathrm{~h}$. Ruxolitinib (Rux) was used as a positive control. The hit compounds that induced the decrease of PD-L1 levels are shown in blue. The depth of blue represents decreased level of PD-L1. The heatmap represents the targeted pathways obtained from the high-throughput screening, based upon decreased membrane PD-L1 level detected by flow cytometry. b Immunoblotting of PD-L1 in U937 cells treated with ES-072 (ES) or AZD9291 (AZD) at indicated concentrations for $12 \mathrm{~h}$. ES and AZD are EGFR inhibitors. c, d Median

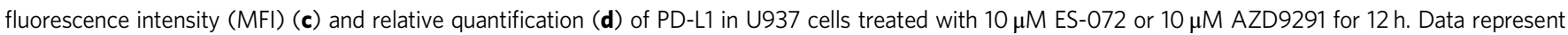
means \pm SEM, $n=18,6$ independent repeats, ${ }^{\star \star \star} P<0.0001$. e-g Immunoblotting (e, $\mathbf{f}$ ) and flow cytometry (g) analysis of PD-L1 levels in U937 cells treated with $10 \mu \mathrm{M}$ ES-072 or $10 \mu \mathrm{M}$ AZD9291 for the indicated times. h, i Immunoblottings (h) of PD-L1 and $\beta$-TrCP, relative quantification (i) of PD-L1 MFI in H1975 cells transfected with non-targeting siRNA (si-CTRL) or $\beta$-TrCP siRNAs ( $\mathrm{si}-\beta$-TrCP) and treated with or without $10 \mu \mathrm{M}$ ES-072/AZD9291 for $12 \mathrm{~h}$. Data represent means \pm SEM, $n=9,3$ independent repeats, NS: no significant; ${ }^{\star \star \star \star} p<0.0001$. j-I Immunoblotting (j) and flow cytometry analysis ( $\mathbf{k}$ ) with relative quantification (I) of PD-L1 in H1975 cells treated with $10 \mu \mathrm{M}$ ES-072 or AZD9291, and/or $5 \mu$ M LY2090314 (LY) for $12 \mathrm{~h}$. LY is a GSK3 inhibitor. Data represent means \pm SEM, $n=9,3$ independent repeats, ${ }^{\star \star \star \star} P<0.0001$. Source data are provided as a Source Data file.

T790M, and T790M/L858R EGFR with IC50 values of $8.9,<0.5$, and $1.75 \mathrm{nM}$, respectively (Supplementary Table 3 ). Furthermore, the decrease of PD-L1 levels induced by ES-072 is dependent on EGFR (Supplementary Fig. 3h). These results suggest that the effect of ES-072 on PD-L1 is via EGFR, consistent with the notion that ES-072 treatment targets PD-L1 for proteasomal degradation, whereas EGF treatment decreased the basal level of these ubiquitination events (Supplementary Fig. 3i). Together, these data indicated that ES-072 induced the decrease of PD-L1 through inhibition of EGFR, induction of K48-linked ubiquitination, and proteasomal degradation.

PD-L1 degradation depends on its phosphorylation at Ser279 and Ser283. To determine the mechanism behind ES-072-induced
PD-L1 degradation, we tested whether PD-L1 undergoes any phosphorylation changes following treatment with this EGFR inhibitor. As judged by mass spectrometry, ES-072 induced several PD-L1 phosphorylation events (Fig. 2a-c and Supplementary Table 4). The top two most robustly induced phosphorylation events on the cytosolic side of PD-L1 were on Ser279 and Ser283 (Fig. 2a, b and Supplementary Table 4). It should be noted that both Ser279 and Ser283 are located in the intracellular segment of PD-L1 (Fig. 2c). Mutating these residues to the phosphorylation-resistant alanine showed that both of the sites play an important role in ES072-induced PD-L1 degradation, and that dual S279A; S283A (2SA) mutation blocks PD-L1 degradation more potently than either S279A or S283A alone (Fig. 2d). Consistently, chase experiments using protein synthesis inhibitor cycloheximide showed that these 


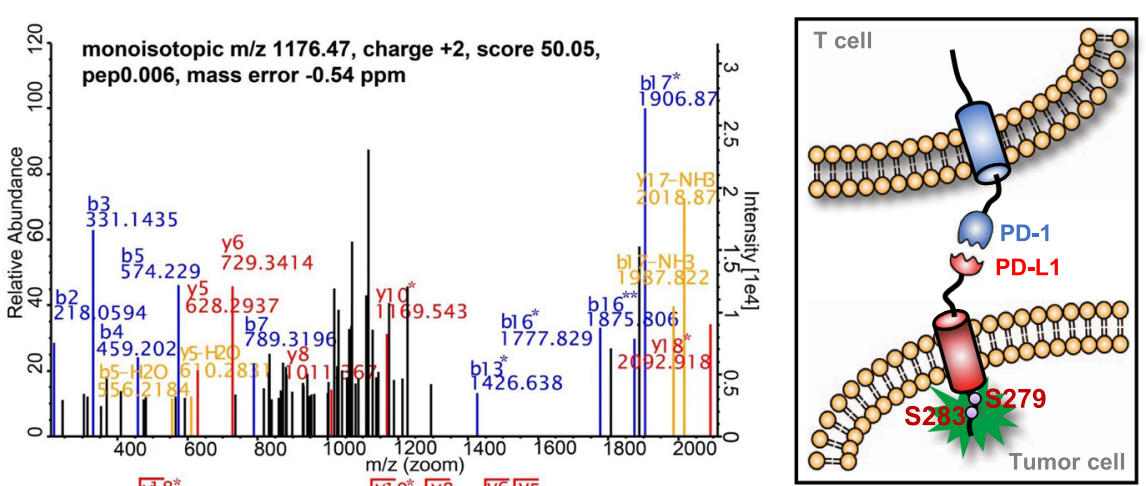

b
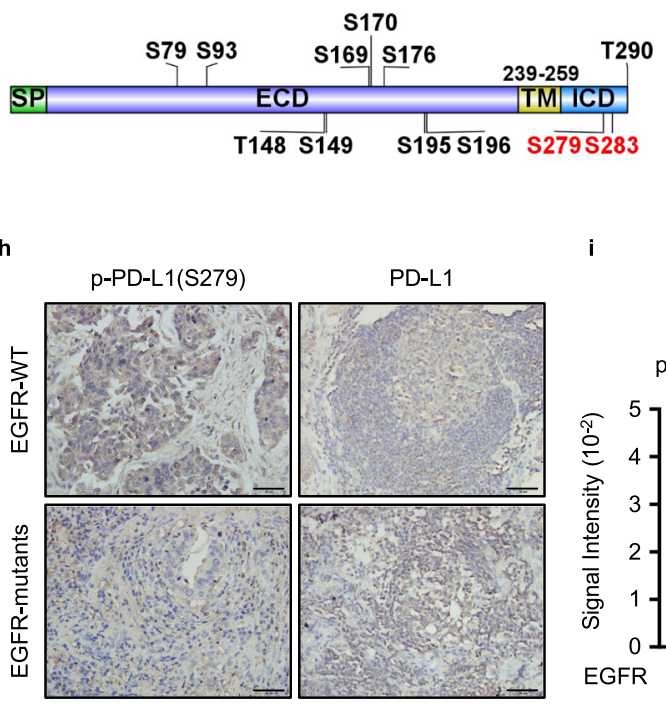

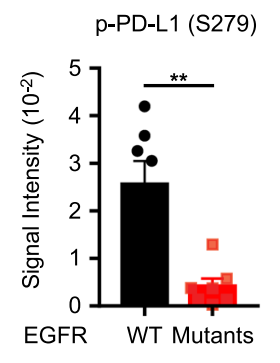

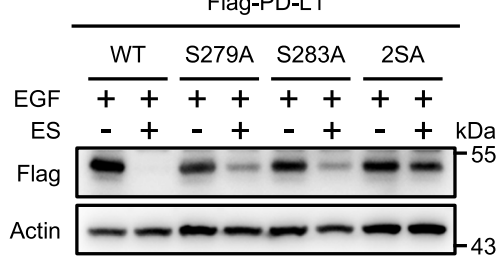

j

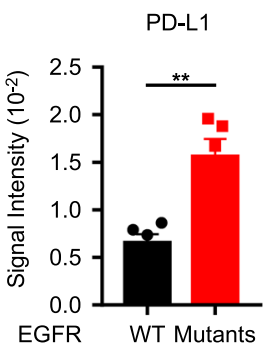

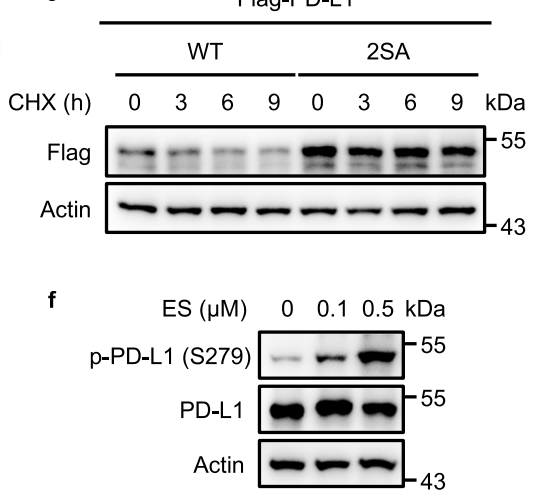

g

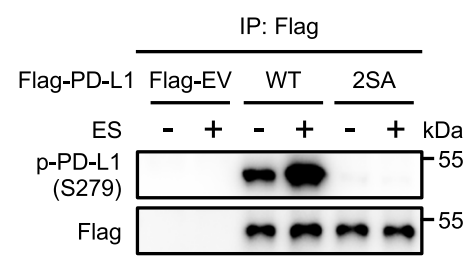

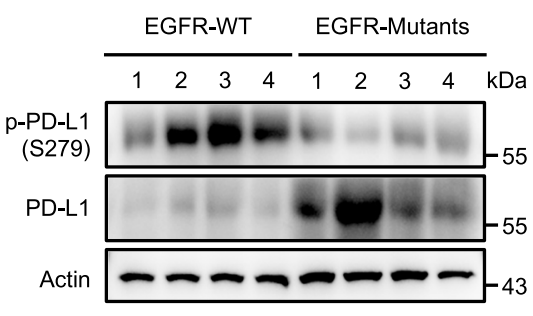

Fig. 2 Phosphorylation on S279/S283 impairs the stability of PD-L1. a Mapping PD-L1 phosphorylation sites following ES-072 treatment. HEK293T cells were transfected with Flag-PD-L1 and treated with $10 \mu \mathrm{M}$ ES-072 for $48 \mathrm{~h}$. Immunoprecipitated PD-L1 was analyzed by mass spectrometry following phosphopeptide enrichment. Peptide ionization data corresponding to Ser279/283 are shown. b Schematic diagram of phosphorylated sites on PD-L1. Fulllength PD-L1 was separated into an extracellular domain (ECD) and intracellular domain (ICD). SP: signal peptide; TM: transmembrane domain. c Schematic diagram of phosphorylated sites, S279 and S283 in the ICD of PD-L1. Positions of phosphorylated sites were labeled in red. This image was created by the first author. d Immunoblots of PD-L1 (anti-Flag) in HEK293T cells transfected with Flag-PD-L1 (WT) or Flag-PD-L1 (mutants) following treatment with 25 $\mathrm{ng} / \mathrm{mL}$ EGF and/or $10 \mu \mathrm{M}$ ES-072 for 48 h, 2SA represents S279A/S283A. e Immunoblots of PD-L1 (anti-Flag) in HEK293T cells transfected with Flag-PD-L1 (WT/2SA) following treatment with $20 \mu \mathrm{g} / \mathrm{mL}$ cycloheximide (CHX) for indicated times. f Immunoblots of H1975 cell lysates following ES-072 treatment for $2 \mathrm{~h}$, at indicated doses. $\mathbf{g}$ HEK293T cells were transfected with Flag-PD-L1 (WT/2SA) following treatment with $10 \mu \mathrm{M} \mathrm{ES-072} \mathrm{for} 2 \mathrm{~h}$; PD-L1 was immunoprecipitated with anti-Flag and immunoblotted with indicated antibodies. Flag-tagged empty vector (Flag-EV) was transfected as a negative control. h Representative images of p-PD-L1 (Ser279) and PD-L1 immunohistochemistry (IHC) staining from EGFR wild-type vs. mutant human alveolar adenocarcinoma specimens. Scale bars represent $50 \mu \mathrm{m}$. $\mathbf{i}, \mathbf{j}$ Quantification of IHC analysis for P-PD-L1 (Ser279) (i) and PD-L1 (j) in $\mathbf{h}$. Data represent means $\pm \mathrm{SEM}, n=7$ (i) $, n=5(\mathbf{j}),{ }^{\star \star} P<0.01, P=0.0013(\mathbf{i}) ; P=0.002(\mathbf{j}) . \mathbf{k}$ Immunoblottings of $p-P D-L 1$ (Ser279) and PD-L1 in EGFR wild-type ( $\left.n=4\right)$ vs. mutant $(n=4)$ human lung adenocarcinoma specimens. Source data are provided as a Source Data file.

phosphorylation events are critical for PD-L1 turnover, as the 2SA mutation delayed PD-L1 degradation (Fig. 2e).

To confirm that endogenous PD-L1 is phosphorylated following ES-072 treatment, we generated a phospho-specific antibody against PD-L1 Ser279. Consistent with mass spectrometry data, ES-072 treatment induced robust phosphorylation at Ser279 in both endogenous and recombinant PD-L1, which was blocked by the S279A mutation, indicating the specificity of the antibody (Fig. 2f, g). Notably, immunohistochemistry and western blotting analysis of tumor biopsies obtained from alveolar adenocarcinoma patients showed that PD-L1 phosphorylation levels at Ser279 are higher in EGFR-WT tumors compared to EGFR-mutant-driven tumors, and this difference in phosphorylation levels correlated with lower PD-L1 levels in EGFR-WT tumors (Fig. 2h-k). The EGFR mutations found in these patients were L858R, E542K, P753R, and $\Delta$ ex19, which have been linked to the activation of EGFR and the resistance to EGFR inhibitors in non-small cell lung cancer ${ }^{30}$.

These results indicate that PD-L1 phosphorylation events at Ser279 and Ser283 are regulated downstream of the EGFR signaling pathway and are critical for PD-L1 degradation.

EGFR inhibition-induced PD-L1 phosphorylation at Ser279/ 283 is mediated by GSK3 $\alpha$. GSK3 $\beta$ activation has been previously shown to promote the degradation of PD-L1 downstream 


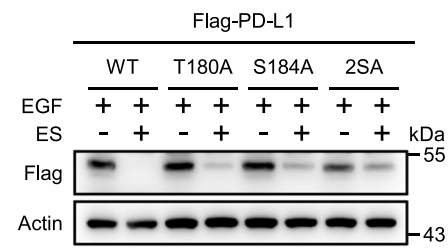

d

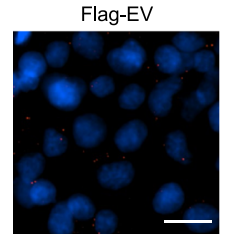

Flag-PD-L1-ES
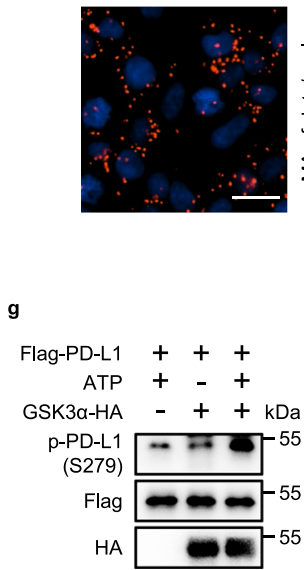

Flag-PD-L1
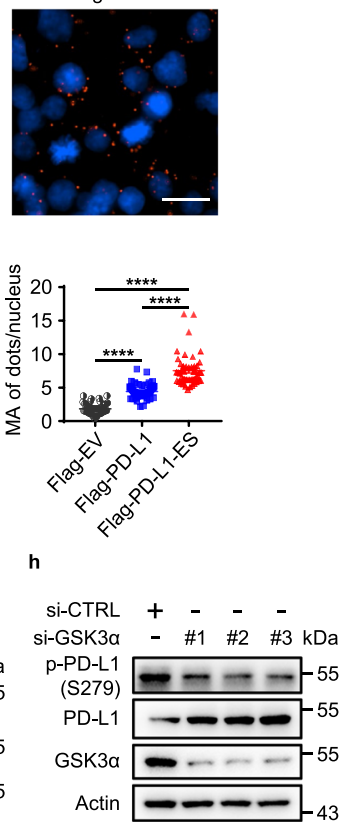

b
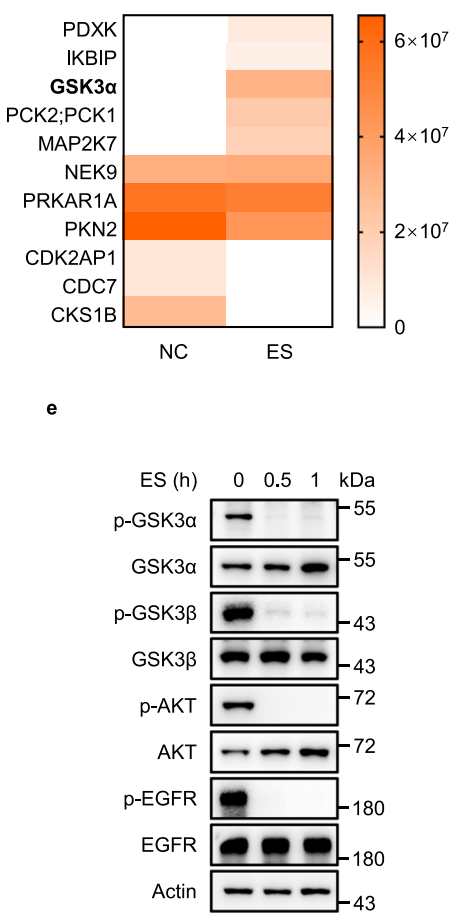

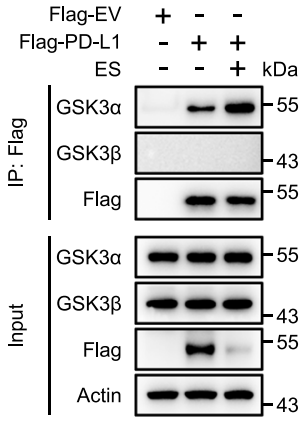

f
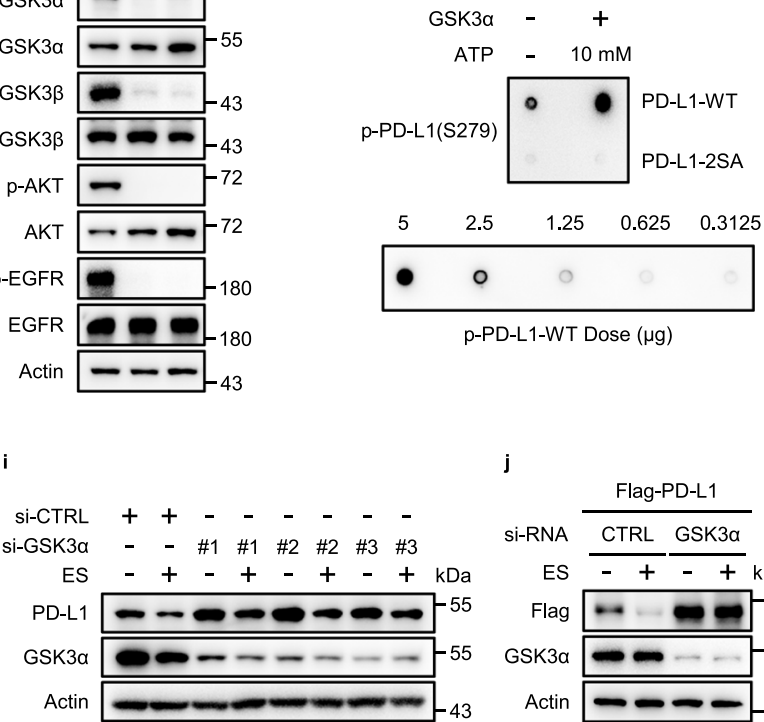

j

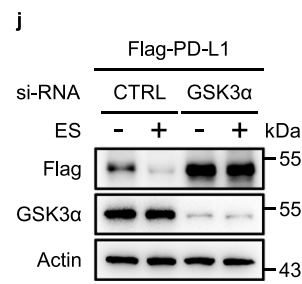

Fig. 3 GSK3 $\alpha$-mediated phosphorylation of PD-L1 promotes PD-L1 degradation. a Immunoblots of PD-L1 (anti-Flag) in HEK293T cells transfected with FlagPD-L1 (WT) or Flag-PD-L1 (mutants) following treatment with $25 \mathrm{ng} / \mathrm{mL}$ EGF and/or $10 \mu \mathrm{M}$ ES-072 for $48 \mathrm{~h}, 2 \mathrm{SA}$ represents T180A/S184A. b HEK293T cells were transfected with Flag-PD-L1 and treated with ES-072 $(10 \mu \mathrm{M})$ for $48 \mathrm{~h}$. Proteins that co-immunoprecipitated (Co-IP) with PD-L1 were analyzed by mass spectrometry. Kinases and kinase-related proteins are shown in heatmap. c Co-IP analysis for the interaction of GSK3 $\alpha / G S K 3 \beta$ and PD-L1 in HEK293T cells transfected with Flag-PD-L1 and treated with or without $10 \mu \mathrm{M} \mathrm{ES-072} \mathrm{for} 12 \mathrm{~h}$, Flag-tagged empty vector (Flag-EV) was transfected as a negative control. d Proximity ligation assay (PLA) analysis for the interaction of GSK3 $\alpha$ and PD-L1 in HEK293T cells treated as c. PLA signals are shown in red and the nuclei in blue; scale bar, $20 \mu \mathrm{m}$. Quantification for the mean area (MA) of PD-L1/GSK3 $\alpha$ PLA speckles is indicated by scattergram. Data represent means \pm SEM, $n=50$, ${ }^{\star \star \star \star \star} P<0.0001$. e Immunoblots of p-GSK3 $\alpha$, GSK3 $\alpha, \mathrm{p}-\mathrm{GSK} 3 \beta, \mathrm{GSK3} \beta, \mathrm{p}-\mathrm{AKT}, \mathrm{AKT}$, p-EGFR, and EGFR in H1975 cells treated with $10 \mu \mathrm{M}$ ES-072 for indicated times. $\mathbf{f}$ In vitro GSK3 $\alpha$ kinase assay was performed in the presence or absence of ATP. The phosphorylation of PD-L1 peptides (PD-L1-WT/2SA) was detected by dot blot with anti-p-PD-L1 (Ser279) antibody, p-PD-L1 peptides were synthesized as a positive control. $\mathbf{g}$ In vitro GSK3 $\alpha$ kinase assay was performed in HEK293T cells transfected with Flag-PD-L1 and GSK3 $\alpha$-HA. Total cell lysates were immunoprecipitated with anti-Flag or anti-HA. The phosphorylation of PD-L1 by GSK3 $\alpha$ was detected using an anti-p-PD-L1 (Ser279) antibody. $\mathbf{h}$ Immunoblots of p-PD-L1, PD-L1, and GSK3 $\alpha$ in H1975 cells transfected with GSK3 $\alpha$-siRNAs. $\mathbf{i}$ Immunoblots of PD-L1 and GSK3 $\alpha$ in U937 cells transfected with GSK3 $\alpha$-siRNAs and treated with or without $10 \mu \mathrm{M} \mathrm{ES-072} \mathrm{for} 24 \mathrm{~h}$. $\mathbf{j}$ Immunoblots of PD-L1 (anti-Flag) and GSK3 $\alpha$ in HEK293T cells transfected with Flag-PD-L1 following treatment with GSK3 $\alpha$-siRNA and treated with or without $10 \mu M$ ES-072 for $24 \mathrm{~h}$. Source data are provided as a Source Data file.

of EGFR inhibition, because the loss of EGFR activity results in a decrease of AKT activity, which normally activates both GSK3 $\alpha$ and GSK $3 \beta^{31}$. We found that mutating the previously reported GSK3 $\beta$ sites on PD-L1 (T180A and S184A) did not fully block ES-072-induced PD-L1 degradation (Fig. 3a), indicating that ES072 promotes $\mathrm{PD}-\mathrm{L} 1$ degradation independent of the previously reported GSK3 $\beta$-driven mechanism.

To determine the kinase responsible for the Ser279/283 phosphorylation events that drive PD-L1 degradation following ES-072 treatment, we performed a large-scale PD-L1 immunoprecipitation experiment followed by mass spectrometry-based proteomic analysis, which revealed several interactors of PD-L1 (Fig. 3b). These results were also confirmed by western blotting experiments (Fig. 3c). Notably, the top endogenous kinase, the interaction of which with PD-L1 was enhanced by ES-072 treatment, was found to be GSK3 $\alpha$, but not GSK3 $\beta$, despite equal expression levels for GSK3 $\alpha$ and GSK3 $\beta$ in HEK293T cells (Fig. 3b, c). This result was also confirmed by tunicamycin treatment, a specific $N$-linked glycosylation inhibitor, using HEK293T cells with exogenous PD-L1 expression (Supplementary Fig. $4 \mathrm{a}-\mathrm{c}$ ).

We further validated these findings by testing for the ability of ES-072 to induce the interaction between PD-L1 and endogenous GSK3 $a$, using proximity ligation assay (PLA), which showed that there is a basal level of GSK3a/PD-L1 interaction, which is enhanced upon ES-072 treatment (Fig. 3d). Interestingly, Ser279 and Ser283 are also a potential match for the consensus motif of GSK3 (p[S/T]XXXp[S/T]p) (Supplementary Fig. 4d) ${ }^{32,33}$. ES-072 
treatment promoted GSK3 $a$ activation in an EGFR- and AKTdependent manner, as judged by the decrease of phospho-GSK3a at Ser21, which is a marker of GSK3 $\alpha$ inhibition (Fig. 3e). ES-072 showed a higher activation effect on GSK3 $\alpha$ when compared to the other two EGFR inhibitors, as judged by loss of the inhibitory phosphorylation of GSK3a (Supplementary Fig. 4e). These findings suggest that GSK $3 \alpha$ could be the kinase that directly phosphorylates PD-L1 at Ser279/283 following EGFR inhibition by ES-072.

Consistent with this hypothesis, purified GSK3a robustly phosphorylated wild-type, but not 2SA synthetic peptides in vitro, at Ser279, which we used to represent the intracellular region of PD-L1 (Fig. 3f). Furthermore, purified GSK3a also robustly phosphorylated purified PD-L1 in vitro, at Ser279 (Fig. 3g). Consistently, knockdown of GSK3 $\alpha$, but not GSK3 $\beta$, blocked the phosphorylation of PD-L1 at Ser279 (Fig. 3h and Supplementary Fig. 4f). These results indicate that GSK3 $a$ directly phosphorylates PD-L1 at Ser279. In agreement with the data showing that Ser279/Ser283 phosphorylation is important for ES072-induced PD-L1 degradation, and that these sites are phosphorylated by GSK3a, knockdown of GSK3 $\alpha$ in U937 and H1975 cells partially rescued PD-L1 degradation induced by ES072 (Fig. 3i and Supplementary Fig. 4g). Similar results were seen when GSK3a was knocked down in HEK293T cells overexpressed with Flag-PD-L1 (Fig. 3j).

Overall, these findings showed that ES-072 treatment results in inhibition of EGFR and activation of GSK3a, which phosphorylates PD-L1 at its cytosolic Ser279 and Ser283 residues to target PD-L1 for proteasomal degradation. Our results also indicate that this mechanism is distinct from the previously reported phosphorylation of PD-L1 by GSK3 $\beta$ at T180 and S184, at the extracellular region of $\mathrm{PD}-\mathrm{L}^{8}$.

E3 ubiquitin ligase ARIH1 directly ubiquitinates PD-L1 and targets it for proteasomal degradation, following EGFR inhibition. To determine which E3 ubiquitin ligase promotes PD-L1 ubiquitination and proteasomal degradation following ES-072 treatment, we analyzed our mass spectrometry immunoprecipitation data for the presence of known mediators of ubiquitination. In addition to cullin ligases, which are known to mediate PD-L1 degradation ${ }^{7,8}$, we found that ES-072 promoted PD-L1 interaction with E3 ubiquitin ligases called ARIH1 and ring finger protein 25 (RNF25) ${ }^{34}$ (Fig. 4a).

We found that transient overexpression of ARIH1 (ARIH1OE), but not that of RNF25, strongly promotes K48-linked ubiquitination of Flag-PD-L1 (Supplementary Fig. 5a). Hemagglutinin (HA)-tagged ARIH1 interacted with endogenous PD-L1 (Fig. 4b) and purified GST-PD-L1 could pull down exogenous ARIH1 from HEK293T cell lysates (Fig. 4c). Importantly, ES-072 treatment enhanced the interaction between ARIH1 and PD-L1 (Fig. 4d), consistent with the mass spectrometry data shown in Fig. 4a. In agreement with the notion that ARIH1 promotes PDL1 degradation, the turnover of PD-L1 in cycloheximide-chased HEK293T cells was exacerbated by ARIH1-OE (Fig. 4e) and was inhibited by knockdown of ARIH1 (Fig. 4f).

Moreover, ARIH1 overexpression in H1975 and HEK293T cells dose-dependently promoted endogenous PD-L1 degradation (Fig. 4g and Supplementary Fig. 5b), whereas ARIH1 knockdown resulted in accumulation of endogenous PD-L1 levels (Fig. 4h and Supplementary Fig. 5c). The ARIH1-induced PD-L1 ubiquitination was blocked by the C357S ligase-dead mutation of $\mathrm{ARIH}^{9}{ }^{9}$, confirming the involvement of the catalytic activity of this E3 ubiquitin ligase (Supplementary Fig. 5d). Consistently, ARIH1 knockdown reduced basal K48-linked PD-L1 ubiquitination, whereas ARIH1 overexpression dramatically increased it
(Fig. 4i). Importantly, ES-072-induced PD-L1 degradation and K48-linked ubiquitination were rescued by ARIH1 knockdown (Fig. 4j, k). Mutational analysis revealed that ARIH1-driven PDL1 ubiquitination could be blocked by Lys/Arg point mutations of K271 and K281, but not that of K263, K270, or K280 (Supplementary Fig. 6a, b). Importantly, purified ARIH1 ubiquitinated purified PD-L1 in vitro and this was blocked by C357S ligase-dead mutation of ARIH1 (Fig. 41 and Supplementary Fig. 5e).

These results indicate that ARIH1 directly ubiquitinates PD-L1 and targets it for proteasomal degradation, following ES-072induced inhibition of EGFR.

Phosphorylation of PD-L1 at Ser279/283 mediated by GSK3a promotes PD-L1/ARIH1 interaction and subsequent PD-L1 ubiquitination and degradation. We next tested whether GSK3 $\alpha$-mediated PD-L1 phosphorylation at Ser279/283 is the mechanism of recruitment of ARIH1 to PD-L1. HEK293T cells stably expressing ARIH1-HA were transfected with wild-type PD-L1 or the phosphorylation-resistant S279A, S283A, and 2SA mutants. Remarkably, PD-L1 ubiquitination induced by ARIH1 overexpression was partially reduced by the S279A and S283A mutations, and strongly inhibited by the 2SA double mutation of these GSK3a phosphorylation sites (Fig. 5a). In accord with this, ARIH1 interaction with PD-L1 was strongly reduced by the 2 SA mutation (Fig. 5a, HA blot in the IP-Flag-PDL1 panel).

Importantly, ES-072 treatment enhanced the interaction between ARIH1 and wild-type PD-L1 WT, but not that of PDL1 2SA mutant (Fig. 5b), whereas overexpression of GSK3a induced PD-L1 ubiquitination, which was blocked by ARIH1 knockdown (Fig. 5c). Notably, GSK3a inhibitor LY2090314 rescued the ARIH1-overexpression-induced degradation of $\mathrm{PD}$ L1 (Fig. 5d), as well as reduction of its membrane levels (Fig. 5e, f) and K48-linked ubiquitination (Fig. 5g).

These findings suggest that PD-L1 degradation is mediated by its GSK3a-driven phosphorylation at Ser279/283 and subsequent recruitment of ARIH1 to ubiquitinate PD-L1 via K48-linked ubiquitin chains that target it for proteasomal degradation.

ARIH1 promotes anti-tumor immunity via PD-L1 degradation. Immunohistochemistry analysis of biopsies obtained from healthy volunteer lung tissues (control) and lung cancer patients showed that, although protein levels of PD-L1 and phosphoGSK3a (i.e., inhibited GSK3a) were strongly elevated in tumor tissues, ARIH1 protein levels were higher in control samples (Supplementary Fig. 7). This finding is consistent with our discovery that ARIH1 promotes PD-L1 degradation and suggest that loss of ARIH1 expression in cancer is a mechanism of PD-L1 accumulation that drives escape from anti-tumor immunity. The mechanisms that result in decreased ARIH1 expression levels in cancer remain to be elucidated.

Notably, ARIH1 mutation found in large cell lung carcinoma $(\mathrm{Y} 392 \mathrm{C})^{35}$ blocked ARIH1-induced PD-L1 ubiquitination and degradation (Fig. 6a, b), indicating that mutational inactivation of ARIH1 in cancer could lead to accumulation of PD-L1, to promote escape from anti-tumor immunity. This observation suggests a potential mechanism of why this mutation occurs in clinical patients with large cell lung carcinoma.

We established a 4T1 cell line with stable ARIH1-OE to evaluate the role of ARIH1 in PD-L1 degradation and tumorigenesis. In accord with our previous findings, ARIH1-OE reduced the protein levels of PD-L1 (Fig. 6c). ARIH1 overexpression had no effect on cell proliferation in vitro (Supplementary Fig. 8a) and on tumor growth in immunodeficient nude 


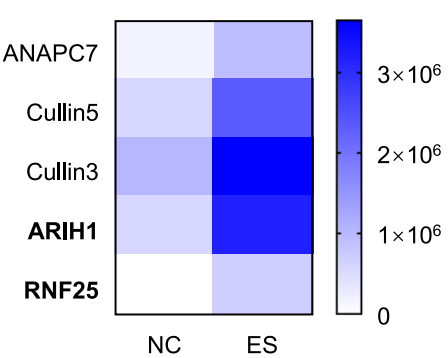

b

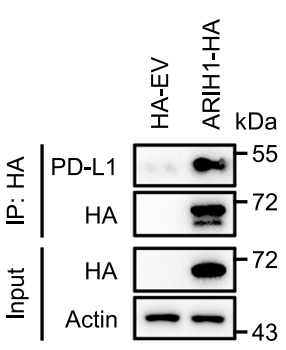

c

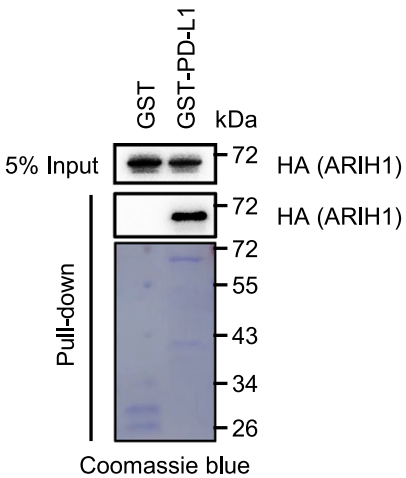

d

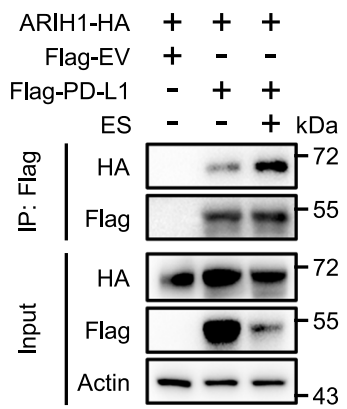

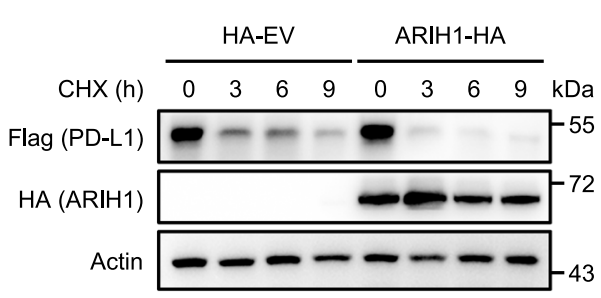

f

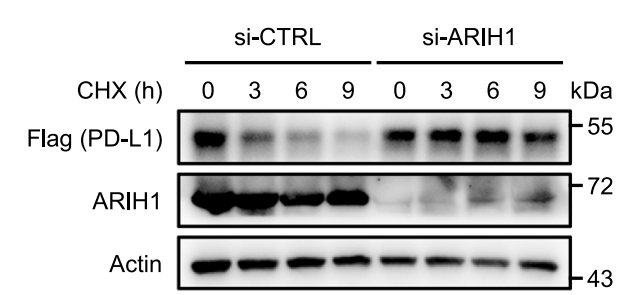

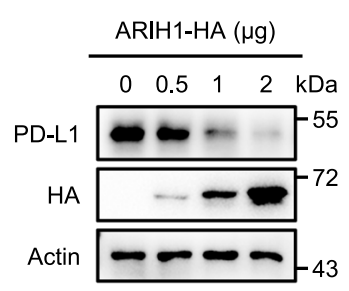

h

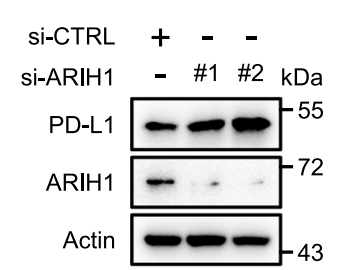

i

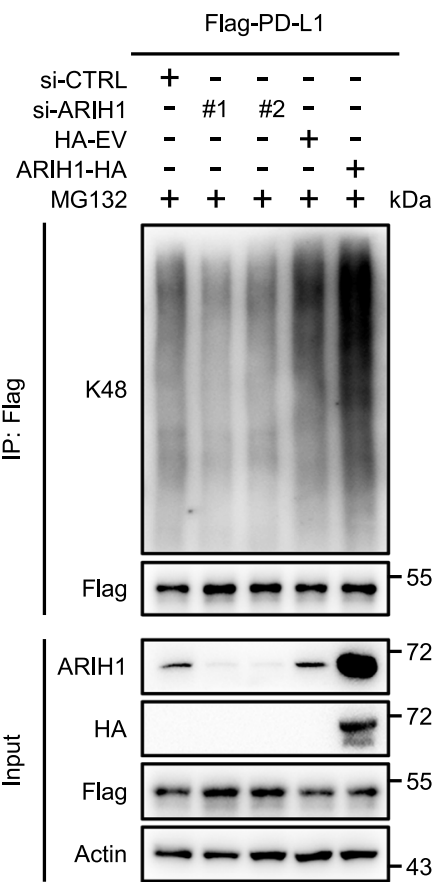

j

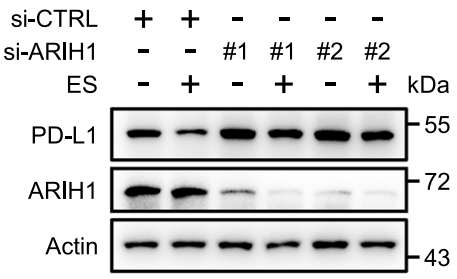

$\mathbf{k}$

I

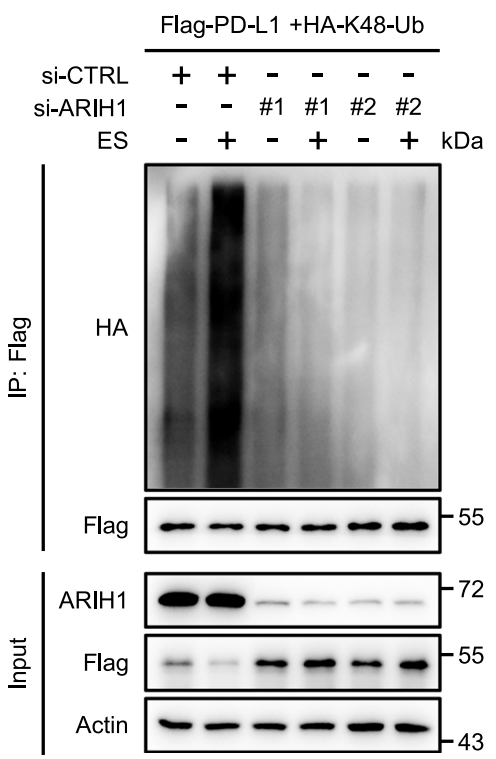

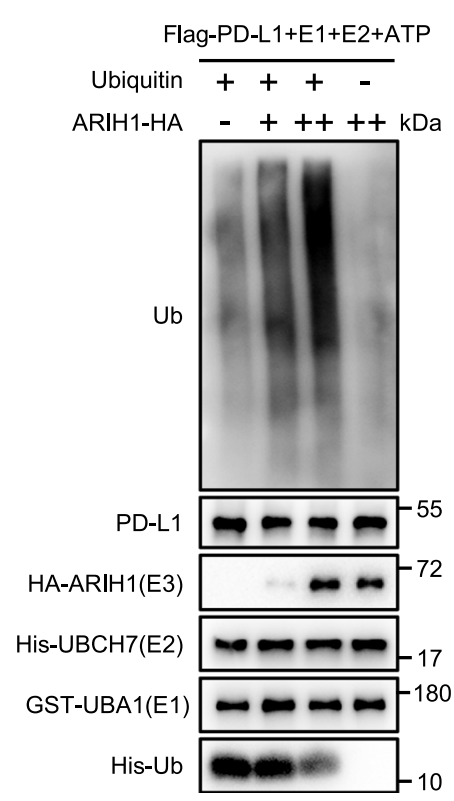

mice (Supplementary Fig. 8b-d). However, we observed a dramatically suppressed tumor growth in the ARIH1-OE group in immunocompetent $\mathrm{BALB} / \mathrm{c}$ mice, the majority of which exhibited a complete tumor regression (Fig. 6d-f). The levels of total and activated $\mathrm{CD}^{+}$cytotoxic $\mathrm{T}$ cells $\left(\mathrm{GzmB}^{+}\right)$that infiltrated the tumor microenvironment were significantly increased in the ARIH1-OE group (Fig. 6g, h and Supplementary Fig. 9). Consistently, ARIH1 overexpression resulted in an increased expression of inflammatory cytokines, including IFN $\gamma$, tumor necrosis factor- $\alpha$, and T-cell chemokines $\mathrm{C}-\mathrm{C}$ motif chemokine ligand 5 (CCL-5) and C-X-C motif chemokine ligand 10 (CXCL-10), as judged by quantitative reverse transcription 
Fig. 4 ARIH1 mediates PD-L1 ubiquitination and degradation. a As in Fig. 3b, except ubiquitination-related proteins are shown in the heatmap. b Co-IP analysis for the interaction of ARIH1 and endogenous PD-L1 in HEK293T cells transfected with HA-ARIH1; HA-tagged empty vector (HA-EV) was transfected as a negative control. c Recombinant PD-L1 was purified using a GST pull-down assay and incubated with HEK293T lysates, which were transfected with HA-ARIH1. The interaction between ARIH1 and PD-L1 was detected by Immunoblot assay. d Co-IP analysis for the interaction of ARIH1 and PD-L1 in HEK293T cells transfected with HA-ARIH1 and Flag-PD-L1, treated with or without $10 \mu \mathrm{M} \mathrm{ES-072} \mathrm{for} 24$ h; Flag-tagged empty vector (FlagEV) was transfected as a negative control. e, f PD-L1 level in $20 \mu \mathrm{g} / \mathrm{mL}$ cycloheximide (CHX)-treated HEK293T cells transfected with or without HA-ARIH1 (e) and ARIH1-siRNA (f). g, h Immunoblots of PD-L1 and ARIH1 (HA) in H1975 cells transfected with ARIH1-HA (g) or ARIH1-siRNAs (h). i HEK293T cells were transfected with Flag-PD-L1. Co-IP analysis for the interaction of K48-ubiquitin and PD-L1 in HEK293T cells transfected with ARIH1-siRNAs or HAARIH1 and treated with MG132 (10 $\mu \mathrm{M}$, a proteasome inhibitor) for $6 \mathrm{~h}$. $\mathbf{j}$ Immunoblots of PD-L1 and ARIH1 in U937 cells transfected with ARIH1-siRNAs, following treatment with $10 \mu \mathrm{M}$ ES-072 for $24 \mathrm{~h}$. k HEK293T cells were transfected with HA-K48-ubiquitin and Flag-PD-L1. Co-IP analysis for the interaction of K48-ubiquitin and PD-L1 in HEK293T cells transfected with ARIH1-siRNAs and treated with or without $10 \mu \mathrm{M} \mathrm{ES-072} \mathrm{for} 24 \mathrm{~h}$. I Recombinant PD-L1 and ARIH1 were purified in transfected HEK293T cells, respectively. An in vitro ubiquitination assay of PD-L1 was performed with purified GST-UBA1 (E1), His-UBCH7 (E2) in the presence or absence of ubiquitin or HA-ARIH1 (E3). Source data are provided as a Source Data file.

a

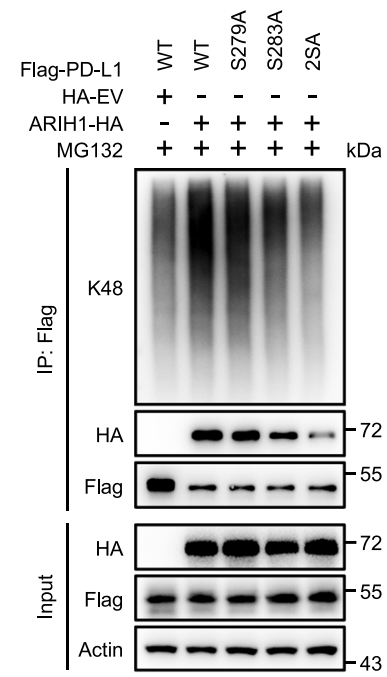

b

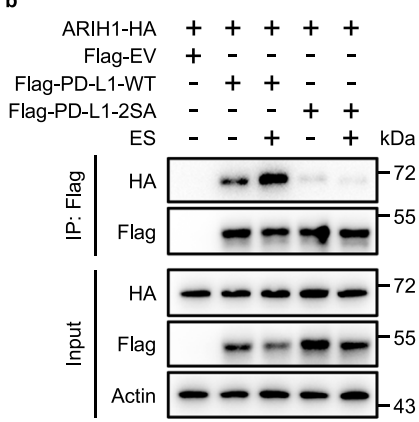

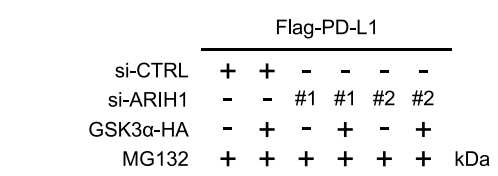
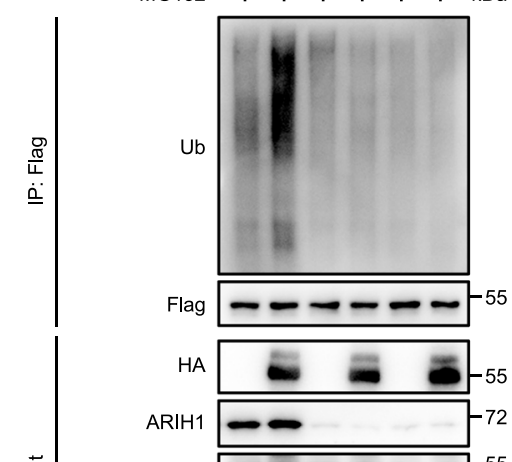

䓂

ARIH

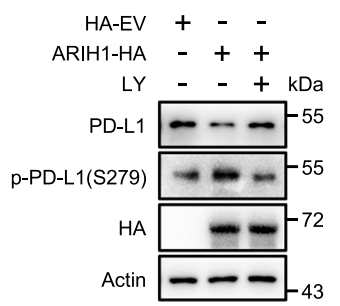

g

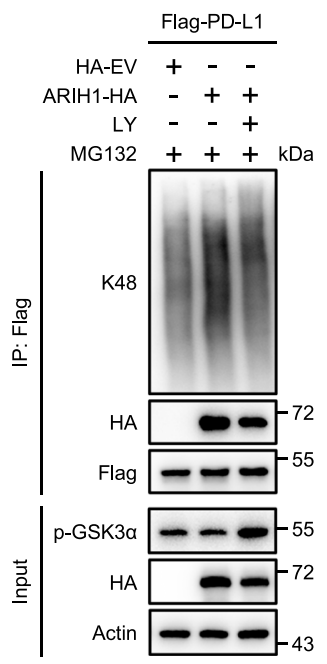

Fig. 5 GSK3 $\alpha$-mediated phosphorylation of PD-L1 promotes PD-L1-ARIH1 interaction and ARIH1-induced degradation. a Co-IP analysis for the interaction of K48-ubiquitin, ARIH1 (HA), and PD-L1 in HEK293T cells transfected with HA-ARIH1 and Flag-PD-L1 (WT, S279A, S283A, or 2SA), treated with MG132 (10 $\mu$ M) for $6 \mathrm{~h}$; HA-tagged empty vector (HA-EV) was transfected as a negative control. b Co-IP analysis for the interaction of ARIH1 and PD-L1 in HEK293T cells transfected with HA-ARIH1 and Flag-PD-L1 (WT or 2SA), treated with $10 \mu \mathrm{M}$ ES-072 for 24 h, Flag-tagged empty vector (Flag-EV) was transfected as a negative control. c HEK293T cells were transfected with Flag-PD-L1. Co-IP analysis for the interaction of ubiquitin and PD-L1 in HEK293T cells transfected with ARIH1siRNAs or HA-GSK3 $\alpha$ and treated with MG132 $(10 \mu \mathrm{M})$ for $6 \mathrm{~h}$; non-targeting siRNA (si-CTRL) was transfected as a negative control. $\mathbf{d}$ Immunoblots of PD-L1 and ARIH1 (HA) in H1975 cells transfected with HA-ARIH1, treated with or without $5 \mu \mathrm{M} \mathrm{LY}$ for 6 h. e, f MFI (e) and relative quantification (f) of PD-L1 in HA-ARIH1overexpressed H1975 cells, treated with or without $5 \mu \mathrm{M}$ LY for $6 \mathrm{~h}$. Data represent means \pm SEM, $n=9,3$ independent repeats, ${ }^{\star \star \star \star} P<0.0001$. $\mathbf{g}$ HEK293T cells were transfected with Flag-PD-L1. Co-IP analysis for the interaction of K48-ubiquitin, ARIH1 (HA), and PD-L1 in HEK293T cells transfected with HA-ARIH1, treated with or without $5 \mu \mathrm{M}$ LY for $12 \mathrm{~h}$ and treated with MG132 $(10 \mu \mathrm{M})$ for $6 \mathrm{~h}$. Source data are provided as a Source Data file. 
a

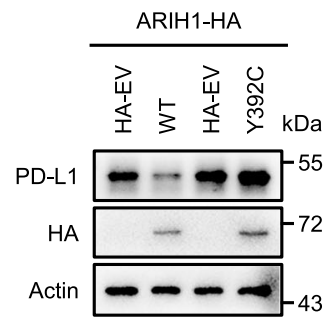

c

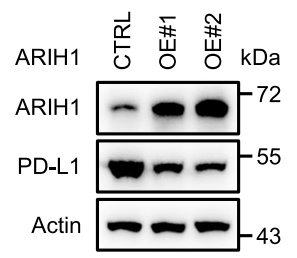

g

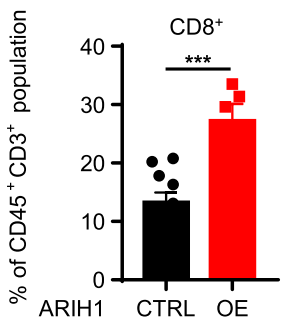

j

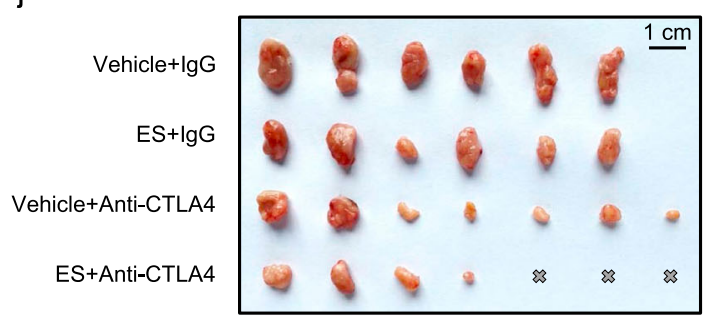

I

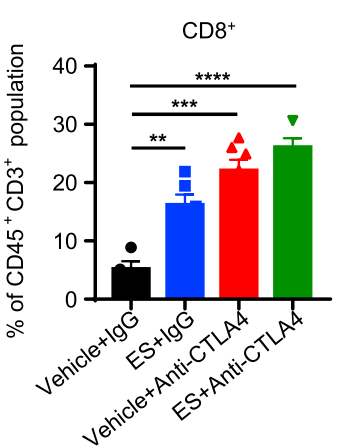

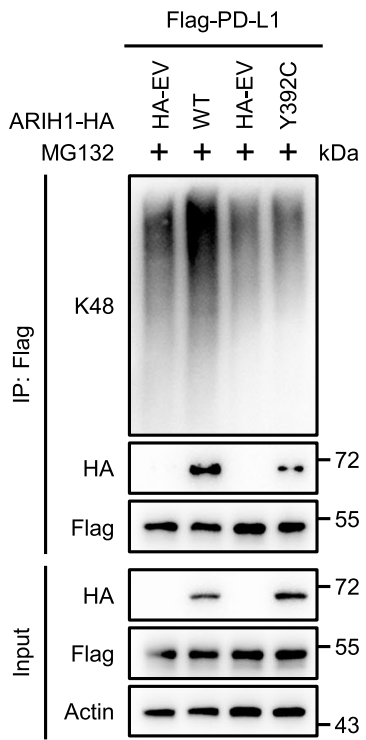

h

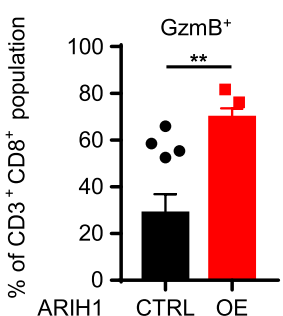

m

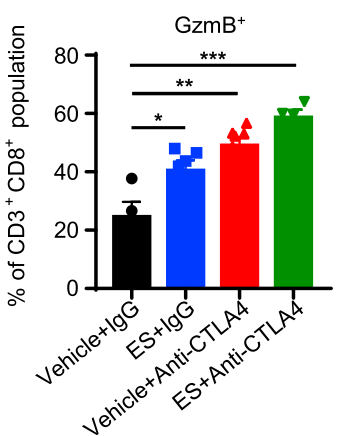

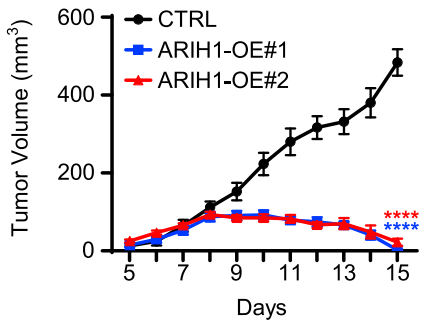

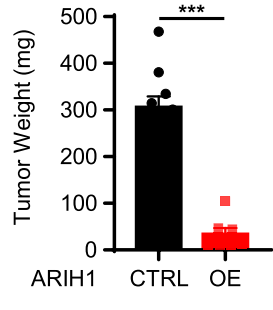

e
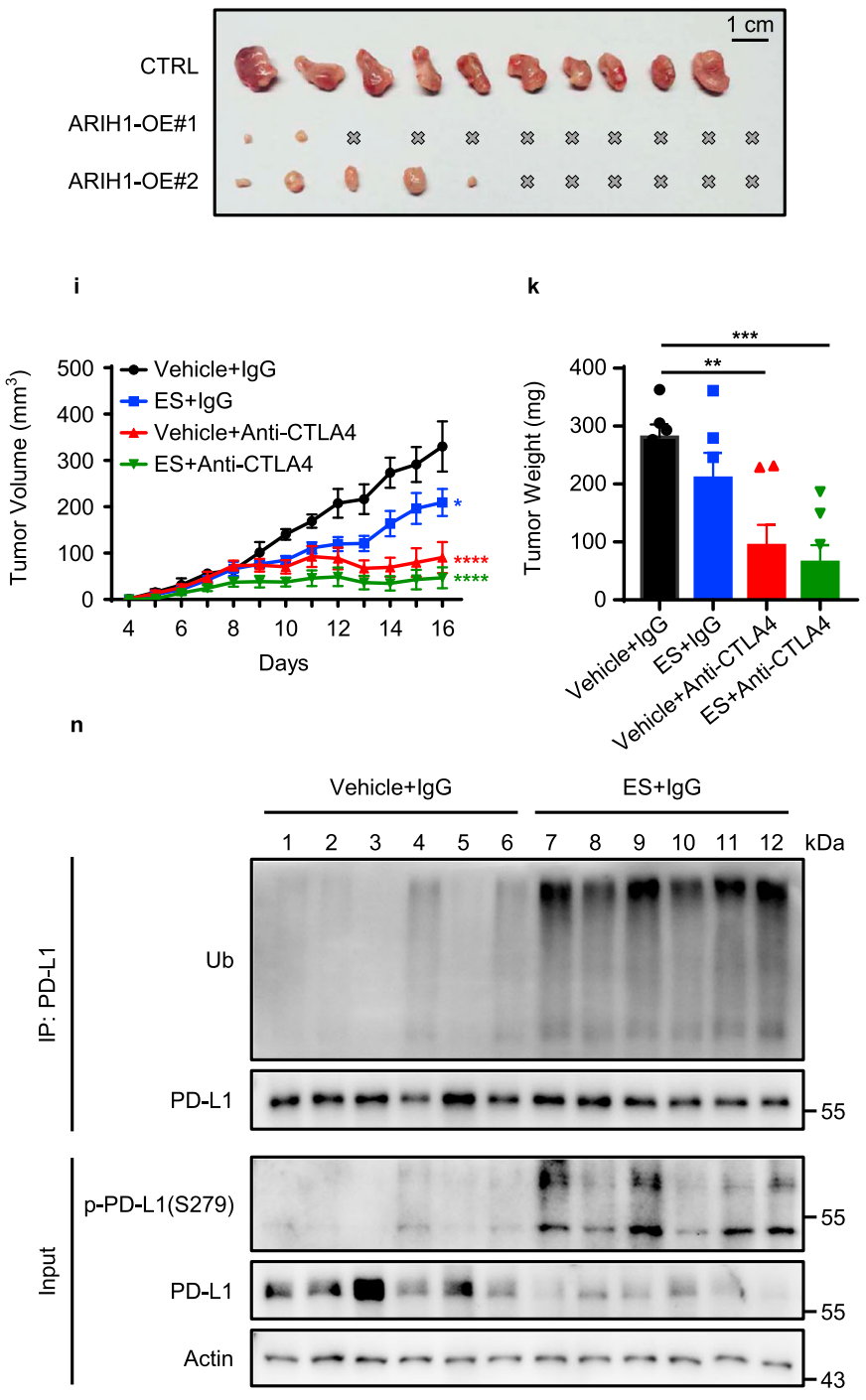

PCR (qRT-PCR) of the whole tumor mRNA (Supplementary Fig. 10a-d).

Recent studies have explored to intercept two immune checkpoint pathways, PD-L1 and CTLA4, collectively, to improve the efficacy of immunotherapy ${ }^{36}$. Next, we used the 4T1 tumor xenograft model and performed an anti-CTLA4 immune checkpoint blockade treatment in the presence/absence of
ES-072. Tumor growth was significantly decreased both by the ES-072 treatment and the anti-CTLA4 treatment. Notably, a further decrease of tumor growth and even complete regression was observed when ES-072 and anti-CTLA4 treatments were combined (Fig. 6i-k). The levels of total and activated CD8 ${ }^{+}$ cytotoxic T cells $\left(\mathrm{GzmB}^{+}\right)$in the tumors were also significantly increased upon the ES-072/anti-CTLA4 combination treatment 
Fig. 6 ARIH1 promotes anti-tumor immunity via PD-L1 degradation. a Immunoblot of PD-L1 and ARIH1 (HA) in H1975 cells transfected with HA-ARIH1 (WT or Y392C); HA-tagged empty vector (HA-EV) was transfected as a negative control. b Co-IP analysis for the interaction of K48-ubiquitin, ARIH1 (HA), and PD-L1 in HEK293T cells transfected with Flag-PD-L1 and HA-ARIH1 (WT or Y392C) in the presence of $10 \mu \mathrm{M} \mathrm{MG132} \mathrm{for} 6 \mathrm{~h}$. c 4 T1 cells were infected with an empty vector (CTRL) or two different ARIH1-overexpressing lentiviral preparations (OE\#1 and OE\#2). ARIH1 and PD-L1 levels were determined by immunoblotting. d-f Tumor growth $(\mathbf{d}, \mathbf{e})$ of CTRL $(n=10)$ and ARIH1-OE cells $(n=11)$ in BALB/c mice and final tumor weights $(\mathbf{f})$. Data represent means $\pm \mathrm{SEM},{ }^{\star \star \star} P<0.001(P=0.0001),{ }^{\star \star \star \star} P<0.0001 . \mathbf{g}$, h Flow cytometry analysis for the tumor levels of CD8 ${ }^{+} \mathrm{T}$ cells $(\mathbf{g})$ and $\mathrm{CD} 8{ }^{+} \mathrm{GzmB}{ }^{+}$ T cells $(\mathbf{h})$. Data represent means \pm SEM, CTRL $(n=10)$ and ARIH1-OE $(n=5)$, ${ }^{\star \star \star} P<0.001(P=0.0006)$, ${ }^{\star \star} P<0.01(P=0.0046)$. i-k 4 T1 tumor xenograft growth in BALB/c mice $(\mathbf{I}, \mathbf{j})$ and final tumor weights $(\mathbf{k})$ following treatment with ES-072 and/or anti-CTLA4 $(n=6-7)$. Vehicle $=$ sodium carboxymethyl (CMC-Na). ES $=$ ES-072. Data represent means $\pm \mathrm{SEM},{ }^{\star} P<0.05(P=0.01),{ }^{\star \star} P<0.01(P=0.0013),{ }^{\star \star \star} P<<0.001(P=0.0001)$,

${ }_{\star \star \star \star \star} P<0.0001$. I, $\mathbf{m}$ Flow cytometry analysis for the tumor levels of CD8 ${ }^{+} \mathrm{T}$ cells $(\mathbf{I})(n=4-7)$ and $\mathrm{CD} 8^{+} \mathrm{GzmB}{ }^{+} \mathrm{T}$ cells $(\mathbf{m})(n=4-7)$. Data represent means \pm SEM. I ${ }^{\star \star} P<0.01(P=0.0017)$, ${ }^{\star \star \star} P<0.001(P=0.0001)$, ${ }^{\star \star \star \star} P<0.0001 . \mathbf{m}{ }^{\star} P<0.05(P=0.0286)$, ${ }^{\star \star} P<0.01(P=0.0012)$, ${ }^{\star \star \star} P<0.001(P=$ 0.00099). $\mathbf{n}$ Immunobloting of the indicated tumor lysates. Source data are provided as a Source Data file.

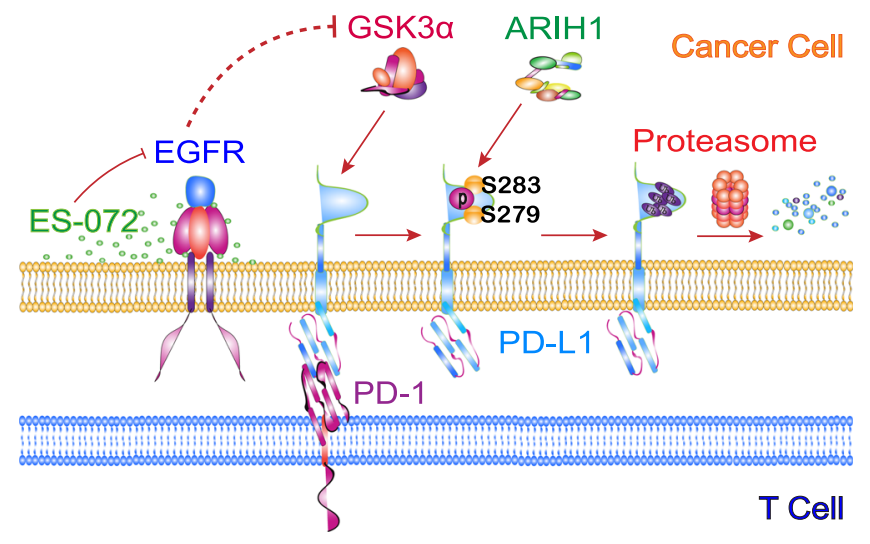

Fig. 7 Schematic model for GSK3 $\alpha$-promoted and ARIH1-mediated PD-L1 degradation. GSK3 $\alpha$ phosphorylates PD-L1 at Ser279 and Ser283. This phosphorylation promotes the binding of PD-L1 with ARIH1, leading to PDL1 ubiquitination and proteasomal degradation. This image was created by the first author.

when compared to either of the single treatments (Fig. 6l, m). Furthermore, western blotting analysis of the tumor lysates showed that ES-072 treatment decreased PD-L1 levels, while increasing its phosphorylation and ubiquitylation levels (Fig. 6n).

Taken together, our data indicate that ARIH1 plays a role in promoting anti-tumor immunity, and that ES-072 is a promising agent to boost anti-tumor immunity and anti-CTLA4 immune checkpoint blockade immunotherapies.

\section{Discussion}

Our study identified that ES-072, an EGFR inhibitor, is an antiPD-L1 agent that induces a signaling cascade downstream of GSK3 $a$ activation. Following ES-072 treatment, GSK3 $a$ phosphorylates PD-L1 at Ser279 and Ser283, which promotes recruitment of the E3 ubiquitin ligase ARIH1 that marks PD-L1 for proteasomal degradation (Fig. 7). Our tumor xenograft experiments revealed an important function of ARIH1 in promoting anti-tumor immunity. This finding indicates that various cancer therapies, including immune checkpoint blockade and cell-based immunotherapies, can be enhanced by supplementation of this inhibitor into the treatment regimens, as not only will it drive EGFR inhibition, decreasing cancer growth, but it will also drive degradation of PD-L1, enhancing anti-tumor immunity.

Recent studies show that PD-L1 expression and degradation levels are regulated by several protein tyrosine kinases, which seem to converge on the PI3K-AKT-GSK3 pathway, yet the mechanisms of PD-L1 degradation regulation were not fully clear ${ }^{37-39}$. Our findings suggest that the EGFR-AKT-GSK3aARIH1 axis is critical for the regulation of PD-L1 degradation.
These results also provide a broad insight into how cancer cells with RTK-activating mutations that drive inhibition of GSK3 may promote escape from anti-tumor immunity by preventing PD-L1 degradation and place RTK inhibitors that result in activation of GSK3 as putative enhancers of the PD-1/PD-L1 immune checkpoint blockade immunotherapies. Our work may pave the way for the augmentation of current immunotherapies that involve targeting PD-1/PD-L1 and help to overcome resistance to such therapies.

GSK3 $\alpha$ and GSK3 $\beta$ share $97 \%$ amino acid similarity in their kinase domains ${ }^{40}$. The isoforms have both unique and overlapping functions, and one isoform cannot completely compensate for the loss function of another one. The most significant evidence for this is that GSK3 $\alpha$-deficient mice show increased insulin sensitivity ${ }^{41}$, whereas GSK3 $\beta$ deletion in mice results in late embryonic death ${ }^{42}$. Growth factors, such as EGF, result in inactivation of GSK3 through Ser9 phosphorylation of GSK3 $a$ or Ser21 phosphorylation of GSK3 $\beta$ by AKT ${ }^{43,44}$.

GSK $3 \beta$ has been reported to promote degradation of PD-L1 via phosphorylation at extracellular T180 and S184, contributing to anti-tumor immunity ${ }^{8}$. These two phosphorylation sites are located in the extracellular region of PD-L1, and their control by GSK3 $\beta$ is not fully understood. In this study, we found that GSK3 $\alpha$, but not GSK3 $\beta$, co-immunoprecipitated with glycosylated PD-L1 following EGFR inhibition by ES-072 (Supplementary Fig. 4a-c). Our mechanistic studies showed that GSK3a phosphorylates PD-L1 at Ser279/283, at the cytosolic region of the receptor (Fig. 2c). The dual-mode of PD-L1 regulation by GSK3 $\alpha$ via its cytosolic region and GSK3 $\beta$ via its extracellular region highlights the importance of oncogenic signaling pathways that converge on the EGFR-AKT-GSK3 $\alpha / \beta$ axis in suppressing PD-L1 degradation and therefore promoting cancer escape from anti-tumor immunity ${ }^{45-47}$. Whether GSK3 $\alpha$ or GSK3 $\beta$ on PD-L1 regulation are the only critical factors remains to be determined.

GSK3 is a tumor suppressor, with important roles in both solid tumors and blood tumors ${ }^{48-51}$, where it regulates cancer cell viability and proliferation ${ }^{52,53}$. Aberrant expression of GSK3a in lung cancer has prognostic significance for clinical treatment ${ }^{54}$. In light of these reports and findings of our study, GSK3-activating agents are likely to promote a strong PD-L1 degradation phenotype and inhibit cancer escape from anti-tumor immunity, thereby enhancing various cancer therapies, including immunotherapies.

We identified ARIH1 as a promoter of anti-tumor immunity via induction of PD-L1 degradation. Previously, ARIH1 was shown to be elevated in head and neck squamous cell carcinoma biopsies ${ }^{55}$. However, we found that in lung alveolar adenocarcinoma biopsies, ARIH1 levels are lower than in control samples. This intriguing cancer-specific difference invites further investigation in future studies, to determine how it relates to the escape 
from each cancer type from anti-tumor immunity. Interestingly, ARIH1 missense mutations are found in $4 \%$ of non-small cell lung cancer patients $(n=75)^{56}, 3.25 \%$ of prostate cancer patients $(n=154)^{57}$, and $5.13 \%$ of cutaneous squamous cell carcinoma patients $(n=39)^{58}$.

In addition to ARIH1, PD-L1 is also targeted for degradation by $\mathrm{Cul}^{\mathrm{SPOP}}$ and $\beta-\mathrm{TrCP}^{7,8} \mathrm{E} 3$ ubiquitin ligases. Together, these findings suggest that combination treatments that target more than one of these E3 ubiquitin ligases may have potentially additive/synergistic effects on PD-L1 degradation in cancer, allowing a more potent stimulation of anti-tumor immunity.

In summary, our study sheds light on the mechanisms of cancer escape from anti-tumor immunity via increased PD-L1 protein levels downstream of EGFR overexpression and overactivation in cancer. Our work suggests that GSK3 $\alpha$ - and ARIH1activating agents, as well as EGFR inhibitors, are likely to stimulate anti-tumor immunity and therefore enhance existing cancer therapies by triggering the PD-L1 degradation pathway this work delineates.

\section{Methods}

Reagents and antibody generation. The compounds and their sources are as follows: S-Ruxolitinib (\#INCB018424; Selleck), AZD9291, Osimertinib (\#S7297; Selleck), MG132 (\#S2619; Selleck), LY2090314 (\#S7063; Selleck), and ES-072 were synthesizedin collaboration with Shanghai Institute of Organic Chemistry, Chinese Academy of Sciences. The recombinant cytokines and their sources are as follows: Human recombinant IFN $\gamma$ (\#300-02; Peprotech), Human recombinant EGF (\#AF-100-15; Peprotech), and Mouse recombinant IL-4 (\#214-14; Peprotech). The following antibodies were used in this study: PE anti-human CD274 (\#329706; 1 : 200; Biolegend), PE anti-mouse CD274 (\#124308; $1: 200$; Biolegend), PE Mouse IgG2b (isotype control) (\#400312; $1: 200$; Biolegend), Zombie Violet $^{\text {Tw }}$ Fixable Viability Kit (\#423114; $1: 200$; Biolegend), PerCP/Cyanine5.5 anti-mouse CD45 (\#103132; 1 : 200; Biolegend), PE/Cyanine7 anti-mouse CD3 (\#100320; $1: 200$; Biolegend), FITC anti-mouse CD8 (\#100706; $1: 200$; Biolegend), APC antihuman/mouse Granzyme B (\#372204; $1: 200$; Biolegend), PD-L1 (ab213524, 1:1000; Abcam), PD-L1 (66248-1-Ig, 1 : 1000; Proteintech), $\beta$-TrCP (D13F10) (\#4394, $1: 1000$ Cell Signaling Technology), EGFR (D38B1, 1:1000; Cell Signaling Technology), Phospho-EGFR (Tyr1068, $1: 1000$; Cell Signaling Technology), Ubiquitin (P4D1) (\#SC8017, 1: 200; Santa Cruz Biotechnology), K48 (05-1307, 1:1000; Milipore), GSK3a (\#4337, 1 : 1000; Cell Signaling Technology), Phospho-GSK3a (Ser21) (\#9631, $1: 1000$; Cell Signaling Technology), GSK3 $\beta$ (Y174) (ab32391, 1:5000; Abcam), Phospho-GSK3 $\beta$ (Ser9) (\#P49841, 1:1000; Cell Signaling Technology), AKT (\#9272, 1:1000; Cell Signaling Technology), Phospho-AKT (Ser473) (\#4046, 1 : 2000; Cell Signaling Technology), ARIH1 (C-7) (\#SC-514551, $1: 200$; Santa Cruz Biotechnology), ARIH1 (Goat) (\#EB05812, $1: 100$; Everestbiotech), GST (B-14) (\#SC-138, 1:200; Santa Cruz Biotechnology), Granyzme B (D6E9W) (\#46890, 1:50; Cell Signaling Technology), His-tag (\#66005-1-Ig, 1 : 1000; Proteintech), Flag-tag (0912-1, $1: 2000$; HuaAn Biotechnology), HA-tag (0906-1, 1:2000; HuaAn Biotechnology), and B-Actin (M1210-2, $1: 2000$; HuaAn Biotechnology). The anti-human phospho-PD-L1 Ser279 antibody was raised against the region near Ser279 phosphorylation site of PD-L1. The secondary antibodies for western blotting were used: goat anti-mouse $(1: 20,000$, \#31430, Thermo Fisher Scientific, Ltd) and goat anti-rabbit ( $1: 20,000$, \#31460, Thermo Fisher Scientific, Ltd). The phosphorylated synthetic peptide [QDTNSKKQSDTHLEC] was used for immunization in rabbits. The antibody was generated by GenScript (Nanjing, China). Anti-HA magnetic beads (\#B26202) and Anti-Flag (DYKDDDDK) Affinity Gel (\#B23102) were from Bimake. Lipofectamine 2000 (\#1901433) and Lipofectamine 3000 (\#2067450) were from Invitrogen. Collagenase/hyaluronidase (\#17100-017, Vancouver, BC, Canada) were from Stemcell Technologies and DNase (\#10104159001) were from Sigma. The peptides and their sources are as follows: PD-L1-WT [LRKGRMMDVKKCGIQDTNSKKQSDTHLERT], phosphorylated PD-L1-WT [LRKGRMMDVKKCGIQDTNS (PO3H2) KKQS (PO3H2) DTHLERT], and PD-L1-2SA [LRKGRMMDVKKCGIQDTNAKKQADTHLEET] were synthesized by Zhongtai (Hangzhou, China).

Cell culture. Human histiocytic lymphosarcoma cell line (U937), Non-small cell lung adenocarcinoma cancer cell line (H1975), and Human embryonic kidney cell line (HEK293T) were a gift from J.Y. Yuan (Harvard Medical School, Boston). PDMs were obtained from male BALB/c mice. PDMs, U937, and H1975 cells were cultured in RPMI-1640 (Hyclone, with L-glutamine); HEK293T cells were cultured in Dulbecco's modified Eagle's medium (Hyclone, with L-glutamine, with $4.5 \mathrm{~g} / \mathrm{L}$ glucose, without pyruvate). These media were supplemented with $10 \%$ heatinactivated fetal calf serum (Gibco) and 1\% Penicillin/Streptomycin (Gibco). All cells were cultured at $37^{\circ} \mathrm{C}$ with $5 \% \mathrm{CO}_{2}$. Cells were transiently transfected with DNA using Lipofectamine 2000 or Lipofectamine 3000.

Plasmids construction and RNA interference. Flag-PD-L1 (WT and mutants), GSK3 $\alpha-H A$, and ARIH1-HA (WT and mutants) were amplified by PCR and fused the fragments into pCMV3 via seamless cloning. The constructs and siRNAs were transfected into cell lines (HEK293T, H1975, and U937) with Lipofectamine 2000 or Lipofectamine 3000, according to the the manufacturer's protocol. siRNAs used in this study are provided in Supplementary Table 5.

In vitro high-throughput drug screens. U937 cells $\left(2 \times 10^{5}\right)$ were preincubated with $100 \mu \mathrm{g} / \mathrm{L}$ IFN $\gamma$ for $48 \mathrm{~h}$ and plated in 96-well plates per well (Corning). Then $10 \mu \mathrm{M}$ FDA-approved drugs or drug candidates were added and incubated for $12 \mathrm{~h}$ All compounds were commercially purchased. Treatments were performed twice; each plate contained a negative control (dimethyl sulfoxide) and a positive control (Ruxolitinib). U937 cells were centrifuged for $5 \mathrm{~min}$ at $1000 \times g$ and the supernatant discarded. The collected cells were washed with phosphate-buffered saline (PBS) twice and incubated with PE anti-human $\mathrm{CD} 274$ at $4{ }^{\circ} \mathrm{C}$ for $30 \mathrm{~min}$. Then the incubated U937 cells were washed and resuspended with PBS, and the protein level of membrane PD-L1 reflected by PD-L1-PE median fluorescence intensity (MFI) was determined using flow cytometry analysis. The hit compounds were picked and classified according to the PD-L1-PE-MFI and the targeted pathways.

Western blot analysis. For western blot analysis, cells were collected and washed with PBS, then lysed in radioimmunoprecipitation assay (RIPA) buffer (1\% Triton $\mathrm{X}-100,100 \mathrm{mM}$ Tris- $\mathrm{HCl} \mathrm{pH} 8.8,100 \mathrm{mM} \mathrm{NaCl}, 0.5 \mathrm{mM}$ EDTA). After incubation on ice for $30 \mathrm{~min}$, the lysates were centrifuged at $12,000 \times g$ for $10 \mathrm{~min}$ at $4{ }^{\circ} \mathrm{C}$. The supernatant was collected and the protein concentration was measured by bicinchoninic acid reaction. Protein samples were added with $2 \times$ loading buffer and heated at $100^{\circ} \mathrm{C}$ for $10 \mathrm{~min}$, separated with SDS-polyacrylamide gel electrophoresis (SDS-PAGE), transferred onto polyvinylidene difluoride membranes, and blocked with 5\% non-fat milk in Phosphate Buffered Saline with $0.1 \%$ Tween-20 (PBST) for $1 \mathrm{~h}$ at room temperature. The membranes were probed with the corresponding primary antibodies at $4{ }^{\circ} \mathrm{C}$ overnight and horseradish peroxidaseconjugated secondary antibodies at room temperature for $1 \mathrm{~h}$. Signals were detected using chemiluminescence reagents (\#4AW001-500, 4A649 Biotech, Co.).

Immunoprecipitation. For immunoprecipitation between PD-L1 and GSK3 $\alpha$ / ARIH1/K48, cells were lysed in RIPA buffer (1\% Triton X-100, $100 \mathrm{mM}$ Tris-HCl pH $8.8,100 \mathrm{mM} \mathrm{NaCl}, 0.5 \mathrm{mM}$ EDTA) supplemented with a complete protease inhibitor cocktail (Bimake, added fresh), and mixed with antibodies at $4{ }^{\circ} \mathrm{C}$ for $4 \mathrm{~h}$; protein $\mathrm{A} / \mathrm{G}$ agarose beads were added and incubated at $4{ }^{\circ} \mathrm{C}$ overnight. Beads were washed three times with RIPA buffer and subjected to western blotting.

Mass spectrometry and data analysis. Flag-tagged PD-L1 was overexpressed in HEK293T cells and were trypsin-digested. PD-L1 was immunoprecipitated with beads following immunoprecipitation. The resulting peptides were subjected to the phosphopeptide enrichment using $\mathrm{TiO}_{2}$ beads. The enriched phospho-peptides were analyzed on the Q Exactive ${ }^{\mathrm{Tm}}$ HF mass spectrometer (Thermo Fisher Scientific). The identification and quantification of phosphorylated peptides were done by MaxQuant. The tandem mass spectra were searched against the UniProt human protein database together with a set of commonly observed contaminants. The precursor mass tolerance was set as 20 p.p.m. and the fragment mass tolerance was set as $0.1 \mathrm{Da}$. The 33 cysteine carbamide methylation was set as a static modification and the methionine oxidation, as well as serine, threonine, and tyrosine phosphorylation, were set as variable modifications. The false discovery rate (FDR) at peptide spectrum match level was controlled below $1 \%$.

Duolink $^{\circledR}$ PLA fluorescence analysis. For the interaction between PD-L1 and GSK $3 \alpha / G S K 3 \beta$, the samples were pre-treated with respect to fixation, retrieval, and/or permeabilization. Then the samples were incubated with Duolink ${ }^{\circledR}$ Blocking Solution in a heated humidity chamber for $60 \mathrm{~min}$ at $37^{\circ} \mathrm{C}$. The samples were incubated with diluted primary antibody in a humidity chamber overnight at $4{ }^{\circ} \mathrm{C}$. The samples were washed with $1 \times$ Wash Buffer $A$ at room temperature for $5 \mathrm{~min}$ twice after the primary antibody solution was moved then incubated with diluted PLUS, and MINUS PLA probes $(1: 5)$ in a pre-heated humidity chamber for $1 \mathrm{~h}$ at $37^{\circ} \mathrm{C}$. The samples were washed with $1 \times$ Wash Buffer A at room temperature for 5 min twice after the PLA probes were moved, then incubated with ligation solution in a pre-heated humidity chamber for $30 \mathrm{~min}$ at $37^{\circ} \mathrm{C}$. The samples were washed with $1 \times$ Wash Buffer $\mathrm{A}$ at room temperature for 5 min twice after the ligation solution was moved then incubated with amplification solution in a pre-heated humidity chamber for $100 \mathrm{~min}$ at $37^{\circ} \mathrm{C}$. The samples were washed with $1 \times$ Wash Buffer B for 10 min twice and $0.01 x$ Wash Buffer B for 1 min at room temperature after the amplification solution was moved. The slides were mounted with a coverslip using a minimal volume of Duolink ${ }^{\circledR}$ PLA Mounting Medium with 4, 6diamidino-2-phenylindole analyzed with confocal microscope. The images were collected using Cytation 3 and were analyzed using Gen5 2.0.

Protein purification and in vitro kinase assays. For purification of PD-L1, Flag tagged PD-L1 was transfected into HEK293T cells for $24 \mathrm{~h}$. Cells were lysed in 1 mL of lysis buffer (TAP) (0.5\% NP-40, 1 mM Na$V_{3} \mathrm{VO}_{4}, 20 \mathrm{mM}$ Tris-HCl pH 7.5, 1 $\mathrm{mM} \mathrm{NaF}, 150 \mathrm{mM} \mathrm{NaCl}, 1 \mathrm{mM}$ EDTA) supplemented with a complete protease inhibitor cocktail (Bimake, added fresh), and incubated with anti-Flag magnetic 
beads (after washing the beads with PBS twice) for $6 \mathrm{~h}$ on a rotating wheel at $4{ }^{\circ} \mathrm{C}$. The beads were washed with TAP buffer three times and treated with CIP (\#M0290, Biolabs) at $37^{\circ} \mathrm{C}$ for $30 \mathrm{~min}$. The kinase assays were performed with recombinant human GSK3a proteins (\#Ab42597, Abcam). The purified PD-L1 or synthetic peptides (PD-L1-WT/2SA) were incubated in $30 \mu \mathrm{L}$ of kinase buffer $(25$ $\mathrm{mM}$ Tris-HCl pH 7.5, $5 \mathrm{mM} \beta$-glycerophosphate, $2 \mathrm{mM}$ dithiothreitol (DTT), $0.1 \mathrm{mM} \mathrm{Na}_{3} \mathrm{VO}_{4}, 10 \mathrm{mM} \mathrm{MgCl}$ ) supplemented with phosphatase inhibitor cocktail (\#b15001, Bimake), with or without $100 \mu \mathrm{M}$ ATP for $2 \mathrm{~h}$ at $37^{\circ} \mathrm{C}$. The reactions were stopped by adding SDS-PAGE $2 \times$ loading buffer $(100 \mathrm{mM}$ Tris- $\mathrm{HCl} \mathrm{pH} 6.8$, $20 \%$ glycerol, $4 \%$ SDS, $0.1 \%$ Bromophenol blue $0.2 \mathrm{M} \mathrm{DTT}$ ) and heating at $100{ }^{\circ} \mathrm{C}$ for $10 \mathrm{~min}$. Kinase activity was evaluated by dot blot or western blotting with antiphospho-human PD-L1 Ser279 antibody.

Flow cytometry analysis of membrane PD-L1. For flow cytometric analysis for membrane PD-L1, H1975 or HEK293T cells were collected by centrifugation at $1000 \times g$ for $5 \mathrm{~min}$, incubated with PBS (0.5\% bovine serum albumin) for $10 \mathrm{~min}$ at room temperature. The cells were probed with PE-conjugated PD-L1 antibody (\#329706, Biolegend) and a matched isotype control at $4^{\circ} \mathrm{C}$ for $30 \mathrm{~min}$ in the dark. After washing three times with PBS, the cells were analyzed using flow cytometry (Beckman Coulter Cytoflex) and data were analyzed using CytExpert V2.3 and and FlowJo X software.

GST-pull down. GST-tagged PD-L1 was expressed in Escherichia coli BL21 and purified. HA-tagged ARIH1 was expressed in HEK293T cells and purified using magnetic HA beads. For reaction, purified GST-PD-L1 was first incubated with glutathione-Sepharose $4 \mathrm{~B}$ beads at $4^{\circ} \mathrm{C}$ for $1 \mathrm{~h}$. Then, the HA-tagged protein was added and incubated at $4{ }^{\circ} \mathrm{C}$ for $2 \mathrm{~h}$. The mixture was boiling with $1 \times$ loading buffer for $10 \mathrm{~min}$, then subjected to western blotting.

In vitro ubiquitination assays. Plasmids GST-UBA1 (E1), Flag-PD-L1, HAARIH1 (E3), and ARIH1 inactive mutant were transfected into HEK293T cells. Post transfection, cells were collected and lysed in RIPA Lysis Buffer at $4{ }^{\circ} \mathrm{C}$ for $1 \mathrm{~h}$. Then, the lysates were incubated with indicated beads at $4{ }^{\circ} \mathrm{C}$ overnight. His-tagged protein UBCH7 (E2) ubiquitin was purified by E. coli BL21 expression. Reactions were performed in a $30 \mu \mathrm{L}$ reaction mixture at $37^{\circ} \mathrm{C}$ for $2 \mathrm{~h}$ in the presence of HisUb, E1, E2, E3, Flag-PD-L1, ATP regeneration solution (Enzo Life Sciences) and Ubiquitin Reaction Buffer (Enzo Life Sciences). All reactions were terminated by boiling 10 min with SDS sample buffer and then subjected to western blotting.

Immunohistochemistry. EGFR-WT tumors and EGFR-mutant-driven tumors from primary lung adenocarcinoma tissues were obtained from 8 patients $(4$ cases each group, median age: 60 years old, range from 47 to 81 ) at the Sir Run Run Shaw Hospital, Zhejiang University. The four EGFR mutations are L858R point mutation of exon 21; E542K point mutation of exon 10 and deletion of exon 19 (ex19del 745-750); P753R point mutation of exon 19; L858R point mutation of exon 21. All samples were collected with signed informed consent according to the internal review and ethics boards of Sir Run Run Shaw Hospital. These tissues were rapidly excised, fixed with $4 \%$ paraformaldehyde, and embedded in paraffin for tissue sections ( $5 \mu \mathrm{m}$ thick) and immunohistochemical staining. The primary antibodies used are anti-PD-L1 (1:200), anti-p-PD-L1 (Ser279) $(1: 200)$, anti-p-GSK3a (1: $200)$, and anti-ARIH1 $(1: 200)$. Visualization of cell nuclei was performed with hematoxylin and analysis was done using the Olympus BX61 light microscope.

Generation of ARIH1-OE stable 4T1 cell lines. Briefly, HEK293T cells were transfected with PCDH-CTRL and PCDH-mouse ARIH1 with packaging plasmids. Medium with secreted viruses was collected at 48,72 , and $96 \mathrm{~h}$, and was filtered through $0.45 \mu \mathrm{m}$ filters. Twenty-four hours post infection, the medium was replaced with fresh medium and the infected $4 \mathrm{~T} 1$ cells were selected with $4 \mu \mathrm{g} / \mathrm{mL}$ puromycin for 3 days.

Tumor xenograft experiments. Female $\mathrm{BALB} / \mathrm{c}$ mice or nude mice (aged 8-10 weeks) were purchased from Shanghai SLAC Laboratory Animal, Co., Ltd (Shanghai, China). All the animal experiments were strictly conducted in accordance with the protocols approved by the Ethics Committee for Animal Studies at Zhejiang University, China.

For xenograft model with control or ARIH1-stable $4 \mathrm{~T} 1$ cells, $5 \times 10^{5}$ control or ARIH1-stable 4T1 cells suspended in $50 \mu \mathrm{L}$ PBS and Matrigel $(1: 1 \mathrm{v} / \mathrm{v})$ were injected into the fourth breast fat pad. On days 3-5 after injection, tumor size was measured and calculated by using the formula $1 / 2 \times$ length $\times$ width $^{2}$. Tumor weight was recorded on the day of killing.

For xenograft model with $4 \mathrm{~T} 1$ cells and combination therapy with ES-072 and anti-CTLA4, $5 \times 10^{5} 4 \mathrm{~T} 1$ cells suspended in $50 \mu \mathrm{L}$ PBS and Matrigel $(1: 1 \mathrm{v} / \mathrm{v})$ were injected into the fourth breast fat pad. On days 3 after injection, tumor size was measured and calculated by using the formula $1 / 2 \times$ length $\times$ width $^{2}$. Mice were then randomly divided into control group: $\operatorname{IgG}(100 \mu \mathrm{g} / 100 \mu \mathrm{L}$, intraperitoneal injection, $\mathrm{i}$. p.) and $0.5 \%$ sodium carboxymethyl $(200 \mu \mathrm{L}$, intragastric administration, i.g $)$ treatment; ES-072 treatment group: IgG $(100 \mu \mathrm{g} / 100 \mu \mathrm{L}$, i.p.) and ES-072 $(60 \mathrm{mg} / \mathrm{kg}$, $200 \mu \mathrm{L}$, i.g.); anti-CTLA4 treatment group: anti-CTLA4 (100 $\mu \mathrm{g} / 100 \mu \mathrm{L}$, i.p.) and $0.5 \%$ sodium carboxymethyl (200 $\mu \mathrm{L}$, i.g.); ES-072 and anti-CTLA4 combination group anti-CTLA4 $(100 \mu \mathrm{g} / 100 \mu \mathrm{L}$, i.p.) and ES-072 $(60 \mathrm{mg} / \mathrm{kg}, 200 \mu \mathrm{L}$, i.g.). ES-072 was given daily from 3 days after inoculation and anti-CTLA4 antibody was administered on day 7,10 , and 13 after inoculation with respective control treatment.

Tumor sample preparation and flow cytometry. Excised tumors were digested in collagenase/hyaluronidase and DNase at $37^{\circ} \mathrm{C}$ for $45 \mathrm{~min}$ to make cell suspension with a $45 \mu \mathrm{m}$ filter (BD Bioscience). Then, cells were stained with Percp-Cy5.5conjugated-CD45, PE-Cy7-conjugated-CD8, FITC-conjugated-CD3 antibodies, fixed and permeabilized with a Fix/Perm kit (Biolegend), and finally stained with APCconjugated-GzmB antibody. Data acquisition was performed using flow cytometry (Beckman Coulter Cytoflex) and data were analyzed using CytExpert V2.3 software.

Immunoprecipitation assay with mouse tumor tissues. For immunoprecipitation between PD-L1 and ubiquitin, fresh xenograft tissues were lysed in RIPA buffer (1\% Triton X-100, 100 mM Tris- $\mathrm{HCl} \mathrm{pH} \mathrm{8.8,} 100 \mathrm{mM} \mathrm{NaCl}, 0.5 \mathrm{mM}$ EDTA) supplemented with a complete protease inhibitor cocktail (Bimake, added fresh) and mixed with anti-PD-L1 at $4^{\circ} \mathrm{C}$ for $4 \mathrm{~h}$; protein A/G agarose beads were added and incubated at $4{ }^{\circ} \mathrm{C}$ overnight. Beads were washed three times with RIPA buffer and subjected to western blotting.

qRT-PCR analysis for tumor cytokines. Fresh tumor tissues were lysed and total RNA was extracted using TRIzol ${ }^{\mathrm{TM}}$ Plus RNA Purification Kit (Invitrogen). cDNA was synthesized from purified RNA using the PrimeScript RT reagent Kit (TAKARA, RR047A) according to the manufacturer's instructions. Quantitative PCR was performed using a StepOnePlus Real-Time PCR Systems (ABI). The comparative $\mathrm{Ct}$ method was used for the data analysis and mouse $\beta$-actin mRNA was used as an internal control. The sequences of primers used for qRT-PCR are provided in Supplementary Table 6.

Statistics and reproducibility. Numerical data are presented as means \pm SEM; all data analyses were performed using GraphPad Prism (version 7.0, GraphPad Software, Inc.). Unpaired Student's $t$-test was used to analyze the flow cytometry data. One-way analysis of variance was used to analyze the statistical differences among the groups with $P$-values indicated in the related graphs. The level of statistical significance was set at a $p<0.05$. All assays were carried out at least three independent times with the same results.

Reporting summary. Further information on research design is available in the Nature Research Reporting Summary linked to this article.

\section{Data availability}

The data that support the findings of this study are available from the corresponding author upon reasonable request. The mass spectrometry proteomics data have been deposited to the ProteomeXchange Consortium (http://proteomecentral proteomexchange.org) via the iProX partner repository with the dataset identifier PXD024452. Source data are provided with this paper.

Received: 9 June 2020; Accepted: 12 March 2021; Published online: 20 April 2021

\section{References}

1. Dong, H. et al. Tumor-associated B7-H1 promotes T-cell apoptosis: a potential mechanism of immune evasion. Nat. Med. 8, 793-800 (2002).

2. Dong, H., Zhu, G., Tamada, K. \& Chen, L. B7-H1, a third member of the B7 family, co-stimulates T-cell proliferation and interleukin-10 secretion. Nat. Med. 5, 1365-1369 (1999).

3. Gibbons, J. R. \& Dong, H. Functional expression of programmed death-ligand 1 (B7-H1) by immune cells and tumor cells. Front. Immunol. 8, 961 (2017).

4. Schreiner, B., Bailey, S. L., Shin, T., Chen, L. \& Miller, S. D. PD-1 ligands expressed on myeloid-derived APC in the CNS regulate T-cell responses in EAE. Eur. J. Immunol. 38, 2706-2717 (2008).

5. Liu, X., Yang, Z., Latchoumanin, O. \& Qiao, L. Antagonizing programmed death- 1 and programmed death ligand-1 as a therapeutic approach for gastric cancer. Ther. Adv. Gastroenterol. 9, 853-860 (2016).

6. Ansell, S. M. et al. PD-1 blockade with nivolumab in relapsed or refractory Hodgkin's lymphoma. N. Engl. J. Med. 372, 311-319 (2015).

7. Zhang, J. et al. Cyclin D-CDK4 kinase destabilizes PD-L1 via cullin 3-SPOP to control cancer immune surveillance. Nature 553, 91-95 (2018).

8. Li, C. W. et al. Glycosylation and stabilization of programmed death ligand-1 suppresses T-cell activity. Nat. Commun. 7, 12632 (2016).

9. Scott, D. C. et al. Two distinct types of E3 ligases work in unison to regulate substrate ubiquitylation. Cell 166, 1198-1214 (2016). 
10. Kelsall, I. R. et al. TRIAD1 and HHARI bind to and are activated by distinct neddylated Cullin-RING ligase complexes. EMBO J. 32, 2848-2860 (2013).

11. Wenzel, D. M., Lissounov, A., Brzovic, P. S. \& Klevit, R. E. UBCH7 reactivity profile reveals parkin and HHARI to be RING/HECT hybrids. Nature 474, 105-108 (2011).

12. Von Stechow, L. et al. The E3 ubiquitin ligase ARIH1 protects against genotoxic stress by initiating a 4EHP-mediated mRNA translation arrest. Mol. Cell. Biol. 35, 1254-1268 (2015).

13. Jutten, B. \& Rouschop, K. EGFR signaling and autophagy dependence for growth, survival, and therapy resistance. Cell Cycle 13, 42-51 (2014).

14. Sigismund, S., Avanzato, D. \& Lanzetti, L. Emerging functions of the EGFR in cancer. Mol. Oncol. 12, 3-20 (2018).

15. Weihua, Z. et al. Survival of cancer cells is maintained by EGFR independent of its kinase activity. Cancer Cell 13, 385-393 (2008).

16. Wang, X., Goldstein, D., Crowe, P. \& Yang, J. Next-generation EGFR/HER tyrosine kinase inhibitors for the treatment of patients with non-small-cell lung cancer harboring EGFR mutations: a review of the evidence. Oncotargets Ther. 9, 5461-5473 (2016).

17. Gainor, J. F. et al. EGFR mutations and ALK rearrangements are associated with low response rates to PD-1 pathway blockade in non-small cell lung cancer: a retrospective analysis. Clin. Cancer Res. 22, 4585-4593 (2016).

18. Camidge, D. R., Doebele, R. C. \& Kerr, K. M. Comparing and contrasting predictive biomarkers for immunotherapy and targeted therapy of NSCLC. Nat. Rev. Clin. Oncol. 16, 341-355 (2019).

19. Azuma, K. et al. Association of PD-L1 overexpression with activating EGFR mutations in surgically resected nonsmall-cell lung cancer. Ann. Oncol. 25, 1935-1940 (2014).

20. Zhang, M. et al. PD-L1 expression in lung cancer and its correlation with driver mutations: a meta-analysis. Sci. Rep. 7, 10255 (2017).

21. Chen, N. et al. Upregulation of PD-L1 by EGFR activation mediates the immune escape in EGFR-driven NSCLC: implication for optional immune targeted therapy for NSCLC patients with EGFR mutation. J. Thorac. Oncol. 10, 910-923 (2015)

22. Zhang, N. et al. The EGFR pathway is involved in the regulation of PD-L1 expression via the IL-6/JAK/STAT3 signaling pathway in EGFR-mutated nonsmall cell lung cancer. Int. J. Oncol. 49, 1360-1368 (2016).

23. Horiuchi, M. et al. Interferon-gamma induces AT(2) receptor expression in fibroblasts by Jak/STAT pathway and interferon regulatory factor-1. Circ. Res. 86, 233-240 (2000).

24. Lau, T. S., Chan, K. Y., Cheung, T. H., Yim, S. F. \& Kwong, J. Abstract 648: interferon-gamma induces PD-L1 expression via IFNGR-JAK-STAT pathway in ovarian cancer. Cancer Res. 77, 648 (2017).

25. Chen, M. et al. JAK2 and PD-L1 amplification enhance the dynamic expression of PD-L1 in triple-negative breast cancer. Clin. Breast Cancer 18, e1205-e1215 (2018)

26. Cross, D. A. et al. AZD9291, an irreversible EGFR TKI, overcomes T790Mmediated resistance to EGFR inhibitors in lung cancer. Cancer Discov. 4, 1046-1061 (2014).

27. Ku, B. M. et al. AZD9291 overcomes T790M-mediated resistance through degradation of EGFRL858R/T790M in non-small cell lung cancer cells. Invest. N. Drug. 34, 407-415 (2016).

28. Cheng, C. C. et al. Epidermal growth factor induces STAT1 expression to exacerbate the IFNr-mediated PD-L1 axis in epidermal growth factor receptor-positive cancers. Mol. Carcinog. 57, 1588-1598 (2018).

29. Chen, M. et al. Insluin and epithelial growth factor (EGF) promote programmed death ligand $1(\mathrm{PD}-\mathrm{L} 1)$ production and transport in colon cancer stem cells. BMC Cancer 19, 112-153 (2019).

30. Engelman, J. A. \& Janne, P. A. Mechanisms of acquired resistance to epidermal growth factor receptor tyrosine kinase inhibitors in non-small cell lung cancer. Clin. Cancer Res. 14, 2895-2899 (2008).

31. Kim, J. et al. The effect of Helicobacter pylori on epidermal growth factor receptor-induced signal transduction and the preventive effect of celecoxib in gastric cancer cells. Gut Liver 7, 552-559 (2013).

32. Nicot, A. et al. Phosphorylation of NBR1 by GSK3 modulates protein aggregation. Autophagy 10, 1036-1053 (2014).

33. Xu, C., Kim, N. G. \& Gumbiner, B. M. Regulation of protein stability by GSK3 mediated phosphorylation. Cell Cycle 8, 4032-4039 (2009).

34. Bouchal, P. et al. Combined proteomics and transcriptomics identifies carboxypeptidase $\mathrm{B} 1$ and nuclear factor $\mathrm{\kappa B}(\mathrm{NF}-\mathrm{\kappa B})$ associated proteins as putative biomarkers of metastasis in low grade breast cancer. Mol. Cell. Proteomics 14, 1814-1830 (2015).

35. Abaan, O. D. et al. The exomes of the NCI-60 panel: a genomic resource for cancer biology and systems pharmacology. Cancer Res. 73, 4372-4382 (2013).

36. Cha, J. et al. Metformin promotes antitumor immunity via endoplasmicreticulum-associated degradation of PD-L1. Mol. Cell 71, 606-620 (2018)

37. Kim, S. et al. Alterations in PD-L1 expression associated with acquisition of resistance to ALK inhibitors in ALK-rearranged lung cancer. Cancer Res. Treat. 51, 1231-1240 (2019).
38. Ota, K. et al. Induction of PD-L1 expression by the EML4-ALK oncoprotein and downstream signaling pathways in non-small cell lung cancer. Clin. Cancer Res. 21, 4014-4021 (2015).

39. Balan, M. et al. Novel roles of c-Met in the survival of renal cancer cells through the regulation of HO-1 and PD-L1 expression. J. Biol. Chem. 290 $8110-8120$ (2015).

40. Woodgett, J. R. Molecular cloning and expression of glycogen synthase kinase3/factor A. Embo J. 9, 2431-2438 (1990).

41. Lal, H. et al. Glycogen synthase kinase-3a limits ischemic injury, cardiac rupture, post-myocardial infarction remodeling and death. Circulation 125, 65-75 (2012)

42. Kerkela, R. et al. Deletion of GSK-3 $\beta$ in mice leads to hypertrophic cardiomyopathy secondary to cardiomyoblast hyperproliferation. J. Clin. Invest. 118, 3609-3618 (2008).

43. Matsuda, T. et al. Distinct roles of GSK-3alpha and GSK-3beta phosphorylation in the heart under pressure overload. Proc. Natl Acad. Sci. USA 105, 20900-20905 (2008).

44. Ahmad, F. et al. Cardiomyocyte-specific deletion of Gsk3alpha mitigates postmyocardial infarction remodeling, contractile dysfunction, and heart failure. J. Am. Coll. Cardiol. 64, 696-706 (2014).

45. Abdelhamed, S., Ogura, K., Yokoyama, S., Saiki, I. \& Hayakawa, Y. AKTSTAT3 pathway as a downstream target of EGFR signaling to regulate PD-L1 expression on NSCLC cells. J. Cancer 7, 1579-1586 (2016).

46. Lastwika, K. J. et al. Control of PD-L1 expression by oncogenic activation of the AKT-mTOR pathway in non-small cell lung cancer. Cancer Res. 76, 227-238 (2016).

47. Ahmad, F. \& Woodgett, J. R. Emerging roles of GSK-3 $\alpha$ in pathophysiology: emphasis on cardio-metabolic disorders. Biochim. Biophys. Acta Mol. Cell Res. 1867, 118616 (2020).

48. Dembowy, J., Adissu, H. A., Liu, J. C., Zacksenhaus, E. \& Woodgett, J. R. Effect of glycogen synthase kinase-3 inactivation on mouse mammary gland development and oncogenesis. Oncogene 34, 3514-3526 (2015).

49. Mancinelli, R. et al. Multifaceted roles of GSK-3 in cancer and autophagyrelated diseases. Oxid. Med. Cell. Longev. 2017, 1-14 (2017).

50. Cervello, M. et al. Pivotal roles of glycogen synthase-3 in hepatocellular carcinoma. Adv. Biol. Regul. 65, 59-76 (2017).

51. McCubrey, J. A. et al. GSK-3 as potential target for therapeutic intervention in cancer. Oncotarget 5, 2881 (2014).

52. Banerii, V. et al. The intersection of genetic and chemical genomic screens identifies GSK-3a as a target in human acute myeloid leukemia. J. Clin. Investig. 122, 935-947 (2012).

53. Park, S., Lee, J. W., Herbst, R. S. \& Koo, J. S. GSK-3a is a novel target of CREB and CREB-GSK-3a signaling participates in cell viability in lung cancer. PLos ONE 11, e153075 (2016).

54. MacAulay, K. \& Doble, B. W. Glycogen synthase kinase 3a-specific regulation of murine hepatic glycogen metabolism. Cell Metab. 6, 329-337 (2007).

55. Elmehdawi, F. et al. Human Homolog of Drosophila Ariadne (HHARI) is a marker of cellular proliferation associated with nuclear bodies. Exp. Cell Res. 319, 161-172 (2013).

56. Hellmann, M. D. et al. Genomic features of response to combination immunotherapy in patients with advanced non-small-cell lung cancer. Cancer Cell 33, 843-852 (2018)

57. Kumar, A. et al. Substantial interindividual and limited intraindividual genomic diversity among tumors from men with metastatic prostate cancer. Nat. Med. 22, 369-378 (2016).

58. Pickering, C. R. et al. Mutational landscape of aggressive cutaneous squamous cell carcinoma. Clin. Cancer Res. 20, 6582-6592 (2014).

\section{Acknowledgements}

This work was supported by the National Natural Science Foundation of China (number 81773182, 91854108, 31601121, 81870007, 81920108001, and 81800024), the National Key R\&D Program of China (2017YFA0104200), Zhejiang Provincial Program for the Cultivation of High-Level Innovative Health Talents (2016-63) and Zhejiang Provincia Natural Science Foundation (LD19H160001). We thank Professor Hui Yang from the Institute of Neuroscience, Chinese Academy of Sciences for cDNA, Professor Qiming Sun from Zhejiang University for his helpful suggestions to this project.

\section{Author contributions}

H.X. conceived and coordinated the project. H.X., S.Y., and A.N. designed the experiments interpreted the data, and wrote the manuscript. Y.W., C.Z., X.L., and Z.H. performed most of the experiments and interpreted the data. B.S., Q. Zeng, Q. Zhao, H.Z., H.L., X.C., X.X., M.Z., T.H., Z.W., H.Y., S. Yang, Y.S., Y.C., R.W., T.X., and W.C. assisted with the experiments and helped to analyze the data. H.X., A.N., S. Ying, Y.W., and C.Z. revised the manuscript.

\section{Competing interests}

The authors declare no competing interests. 


\section{Additional information}

Supplementary information The online version contains supplementary material available at https://doi.org/10.1038/s41467-021-22467-8.

Correspondence and requests for materials should be addressed to A.N., S.Y. or H.X.

Peer review information Nature Communications thanks Seung-Oe Lim and the other, anonymous, reviewer(s) for their contribution to the peer review of this work. Peer reviewer reports are available.

Reprints and permission information is available at http://www.nature.com/reprints

Publisher's note Springer Nature remains neutral with regard to jurisdictional claims in published maps and institutional affiliations. (c) (i) Open Access This article is licensed under a Creative Commons Attribution 4.0 International License, which permits use, sharing, adaptation, distribution and reproduction in any medium or format, as long as you give appropriate credit to the original author(s) and the source, provide a link to the Creative Commons license, and indicate if changes were made. The images or other third party material in this article are included in the article's Creative Commons license, unless indicated otherwise in a credit line to the material. If material is not included in the article's Creative Commons license and your intended use is not permitted by statutory regulation or exceeds the permitted use, you will need to obtain permission directly from the copyright holder. To view a copy of this license, visit http://creativecommons.org/ licenses/by/4.0/.

(C) The Author(s) 2021 